\title{
Drivers of reduced ENSO variability in mid-Holocene in a coupled model
}

\author{
Lin Chen ${ }^{1,2,3} \cdot$ Lu Wang $^{1,2,3}$ (i) $\cdot \operatorname{Tim~Li}^{1,3} \cdot \operatorname{Jian} \mathrm{Liu}^{4,5,6}$
}

Received: 3 February 2018 / Accepted: 8 October 2018 / Published online: 24 December 2018

(C) The Author(s) 2018

\begin{abstract}
The drivers of El Niño-Southern Oscillation (ENSO) intensity change during the mid-Holocene (MH) are investigated through employing a coupled model that exhibits excellent performance in simulating the present-day ENSO behaviors. The model shows a 28\% decrease in ENSO intensity in the MH simulation compared to the pre-industrial (PI) simulation, showing an agreement with the ranges indicated by the paleo-proxies. Based on quantitative analyses, we reveal that the changes in the oceanic dynamic processes (including the thermocline, zonal-advection, and Ekman feedback terms, in the order from being most to least important) were the major drivers of the reduced ENSO intensity in the MH. Further diagnosis analyses show that the weakening in all three oceanic dynamic terms could be traced back to the weakened thermocline response to zonal wind stress anomaly in the MH compared to that in the PI. Such weakened thermocline response was due to the relatively flattened meridional structure of ENSO-related interannual anomaly field (e.g., zonal wind stress anomaly field) in the MH, which arose from the strengthening of the mean meridional overturning circulation, namely, the mean Pacific subtropical cell (STC). The process-oriented analyses throughout this study suggest a critical linkage between ENSO intensity change and mean STC change in the MH, through documenting how the mean STC change altered the oceanic dynamic processes and thus drove the ENSO intensity change.
\end{abstract}

Keywords ENSO variability change $\cdot$ Mid-Holocene simulation $\cdot$ Air-sea feedback

\section{Introduction}

The El Niño-Southern Oscillation (ENSO) is one of the most marked interannual variabilities in the climate system (Philander 1990). Although originating from the tropical Pacific, the ENSO has a vital role in influencing

Lu Wang

luwang@nuist.edu.cn

1 Key Laboratory of Meteorological Disaster, Ministry of Education (KLME)/Joint International Research Laboratory of Climate and Environmental Change (ILCEC)/ Collaborative Innovation Center on Forecast and Evaluation of Meteorological Disasters (CIC-FEMD), Nanjing University of Information Science and Technology, Nanjing, China

2 Laboratory for Regional Oceanography and Numerical Modeling, Qingdao National Laboratory for Marine Science and Technology, Qingdao, China

3 International Pacific Research Center, and School of Ocean and Earth Science and Technology, University of Hawaii at Manoa, Honolulu, HI, USA the weather and climate worldwide (e.g., Trenberth et al. 1998; McPhaden et al. 2006). Understanding potential ENSO intensity change in a warming world has profound meaning; yet, how the ENSO behaviors would change in response to continued anthropogenic warming is still vigorously debated (Collins et al. 2010). For instance, with the aid

4 Key Laboratory for Virtual Geographic Environment, Ministry of Education, Jiangsu Provincial State Key Laboratory Cultivation Base of Geographical Environment Evolution, School of Geography Science, Nanjing Normal University, Nanjing, China

5 Jiangsu Center for Collaborative Innovation in Geographical Information Resource Development and Application, Nanjing, China

6 Jiangsu Provincial Key Laboratory for Numerical Simulation of Large Scale Complex Systems, School of Mathematical Science, Nanjing Normal University, Nanjing, China 
of the projections from the state-of-the-art coupled general circulation models (CGCMs), the projected ENSO intensity changes are divergent; and heretofore the ENSO community even has no consensus on the direction of ENSO intensity change (i.e., becoming stronger or weaker) (e.g., Kim et al. 2014a; Chen et al. 2015; Li et al. 2017). Understanding the physical mechanism for the difference in ENSO intensity between the past climate and the modern-day climate is indispensable for testing the existing mechanisms for ENSO change, and should help us understand future ENSO change (e.g., Emile-Geay et al. 2016).

In the MH (6000 years before the present), the Earth climate system underwent substantial changes in both basic state and ENSO variability due to the switch of the orbital parameters, which provides an integrated framework to study how the ENSO activity would respond to the change in external forcing. Heretofore, a large body of proxy archives, such as corals, ice cores, molluscs, and sediments of seafloor and lake, documented that the ENSO variability was approximately $20-79 \%$ weaker in the $\mathrm{MH}$ relative to the present-day climate (e.g., Tudhope et al. 2001; Cobb et al. 2003; Woodroffe and Gagan 2000; McGregor and Gagan 2004; Koutavas and Joanides 2012; Mcgregor et al. 2013; Karamperidou et al. 2015; Emile-Geay et al. 2016), although some studies (Cobb et al. 2013) elucidated the difficulty of detecting the orbital-forced ENSO change in the Holocene because of the internal variability.

In addition to the paleoclimate records, plenty of studies (e.g., Clement et al. 2000; Liu et al. 2000; Kitoh and Murakami 2002; Otto-Bliesner et al. 2003; Brown et al. 2008; Zheng et al. 2008; Chiang et al. 2009; Braconnot et al. 2012; Luan et al. 2012, 2015; Tian and Jiang 2013; An and Choi 2014; Roberts et al. 2014; Emile-Geay et al. 2016; Tian et al. 2017, 2018) turned to coupled ocean-atmosphere models for help. They found a weakening in ENSO intensity emerged from a wide range of model simulations, although the extent of the reduction in ENSO intensity varied across these modeling results.

Based on model simulation results, various mechanisms have been put forward to account for the reduced ENSO variability in the MH. For instance, Clement et al. (2000) proposed that the reduced ENSO variance in the MH is interpreted as a response to the orbital-induced change in the tropical annual cycle, but the model used in their study is an idealized simple model with much less complexity than a fully-coupled GCM. Liu et al. (2000) suggested that the suppressed ENSO intensity in the MH was attributed to the deepened mean equatorial thermocline and the strengthened Asian summer monsoon. In addition to the effect due to the change in annual cycle, Liu et al. (2000) and Timmermann et al. (2007) suggested that the effect due to the nonlinear frequency entrainment also played a role in suppressing ENSO activity in MH. Chiang et al. (2009) argued that the change in extratropical variability might play a role in suppressing the ENSO amplitude in the MH. Through analyzing seven model simulations from the Paleoclimate Modelling Intercomparison Project Phase 2 (PMIP2), Zheng et al. (2008) pointed out that the reduction of ENSO amplitude should be attributed to the strengthened easterly trade wind in the equatorial Pacific. This notion concluded by Zheng et al. (2008) is in agreement with other modeling studies based on a single CGCM, such as Brown et al. (2008) that employed the HadCM3 model and Luan et al. (2012) that used the IPSL-CM4 model.

It is acceptable that the root cause of climate change in the MH compared to modern-day lies in the change in the incoming solar radiation at the top of atmosphere due to the changes in orbital parameters (Berger 1978). In response to the altered insolation, many aspects of the mean background states varied to different extents in the MH (Braconnot 2007a, b). The reduced ENSO amplitude might be linked to these changes in the basic states, such as the zonal gradient of mean sea surface temperature (SST), the mean trade wind, the distribution of mean thermocline, the mean upwelling, and so on (e.g., Otto-Bliesner et al. 2003). It is plausible that most of the aforementioned changes in the mean state could make a positive contribution to the reduced ENSO intensity, yet there is little consensus on what portion of the mean background state changes played an essential role in suppressing the ENSO variability in the $\mathrm{MH}$.

Moreover, a number of ENSO studies suggested that the ENSO involves various air-sea feedback processes and its growth rate is largely determined by the relevant dynamic and thermodynamic air-sea feedback processes (e.g., Li 1997; Jin et al. 2006; Kim and Jin 2011a, b; Chen et al. 2015). The primary thermodynamic air-sea feedback processes associated with ENSO intensity include the shortwave radiation feedback and the latent heat flux feedback (Sun et al. 2009; Lloyd et al. 2012; Chen et al. 2013, 2018a, b). The main dynamic feedback processes include the thermocline feedback, zonal-advection feedback, and Ekman feedback (e.g., Li 1997; An and Jin 2001; Li et al. 2015a, b, 2017). As described above, many studies argued that some aspects of the mean state changes could affect the ENSO intensity, but few studies clearly explained the specific physical linkage between the mean state change and ENSO intensity change. Therefore, it is necessary to identify which air-sea feedback change plays a dominant role in suppressing the ENSO intensity in the $\mathrm{MH}$, and to further explore the linkage between the key air-sea feedback change and the orbital-driven mean state change in the $\mathrm{MH}$.

This study aims to investigate the following issues. (1) Which change in the dynamic and thermodynamic air-sea feedback processes played a dominant role in reducing the ENSO variability in the MH? (2) What caused the change in the key air-sea feedback processes? (3) How did the changes in the 
ENSO variability and associated air-sea feedback processes link to the orbital-induced mean state changes? Motivated by these questions, we applied the Bjerknes stability index (BJ index; for details, please see Sect. 2.2) to quantitatively diagnose the changes in ENSO intensity and its relevant air-sea feedback processes in the $\mathrm{MH}$, and to reveal the physical mechanisms behind ENSO intensity change. It is worth mentioning that the $\mathrm{BJ}$ index, as a relatively new approach, has been widely employed as an effective tool in some recent studies to address the issues associated with ENSO intensity change (e.g., Kim et al. 2014a; Liu et al. 2014; Wang et al. 2018).

To address the above questions, we conducted a modeling study because model outputs cover nearly all ENSO-related three-dimensional fields, and these outputs have high spatial and temporal resolutions relative to some paleoclimate proxy datasets. We chose a state-of-the-art CGCM, namely, the FGOALS-g2, to conduct the detailed analysis. The FGOALSg2 participated in the PMIP Phase 3 (PMIP3) and the Coupled Model Intercomparison Project Phase 5 (CMIP5). It was chosen here for two reasons. First, the FGOALS-g2 exhibits outstanding performance in representing ENSO characteristics in the present-day simulation among both CMIP Phase 3 (CMIP3) and CMIP5 models (e.g., Chen et al. 2013; Bellenger et al. 2014; Li et al. 2015a, b). Second, the paleoclimate records elucidated that the extent of the reduced ENSO intensity ranged approximately from 20 to 79\%, but previous modeling studies (Zheng et al. 2008; McGregor et al. 2013; An and Choi 2014; Emily-Geay et al. 2016) reported that nearly all the PMIP2 and PMIP3 models severely underestimated the reduction of ENSO intensity (less than 20\%). In contrast, the FGOALS-g2 is the rare CGCM that reproduced a significantly reduced ENSO intensity in the MH (Fig. 1). The magnitude of the reduction of ENSO intensity reached as much as 28-29\% in FGOALS-g2 (Table 1), which is closer to that indicated by the paleoclimate records than those of the other CGCMs. Both reasons gave us confidence to employ FGOALS-g2 as a representative model to investigate the drivers of the reduced ENSO variability in the MH.

The paper is organized as follows. In Sect. 2, we provide a description about the coupled model, the experiments, and method used in the study. We present the changes in the ENSO intensity and the BJ index in Sect. 3. The cause of the weakened oceanic dynamic terms and its linkage to the mean state change are analyzed in Sect. 4. Finally, conclusions and discussion are given in Sect. 5.

\section{Model, data, and method}

\subsection{Brief introduction of FGOALS-g2 and experimental design}

The CGCM employed in this study is the FGOALS-g2 (Li et al. 2013a), which participated in the PMIP3 and CMIP5.

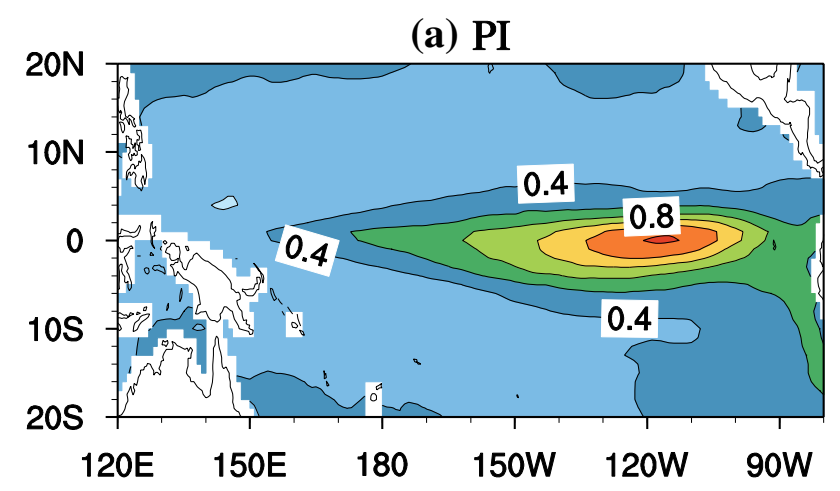

(b) $\mathrm{MH}$

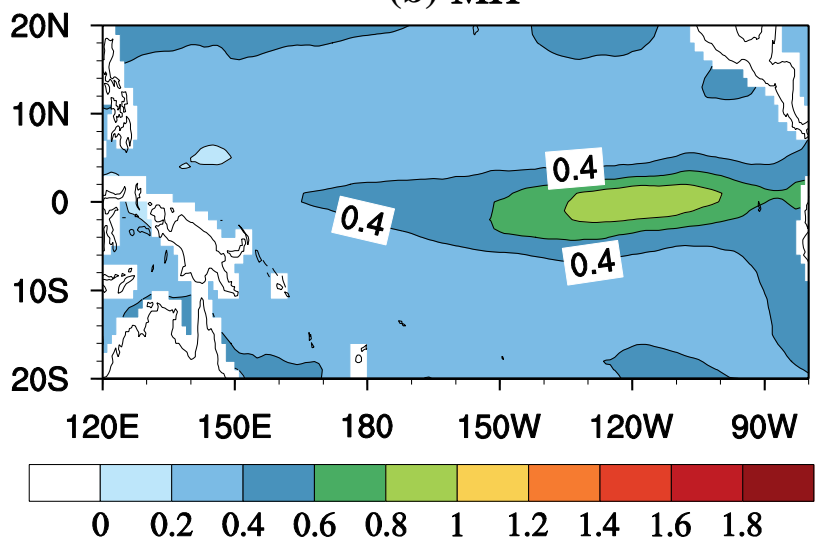

(c) Change

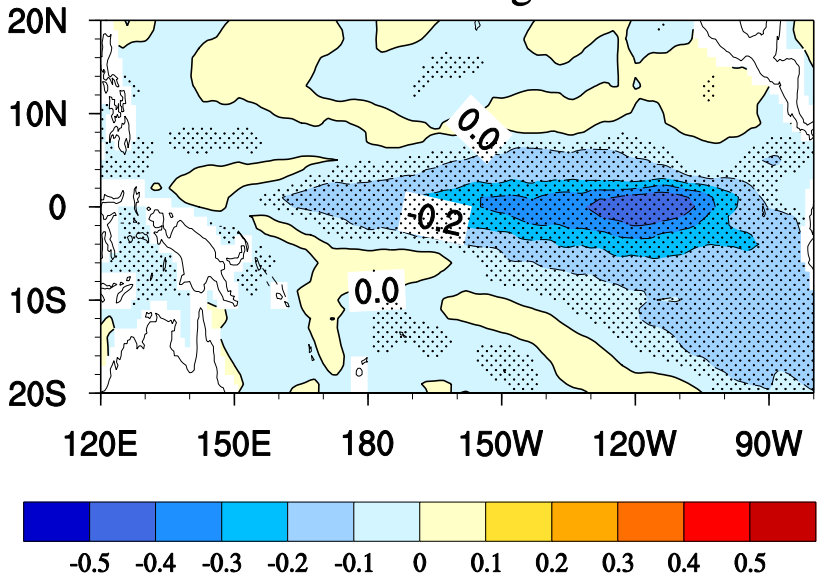

Fig. 1 The standard deviation (Stddev) of SSTA (units: K), derived from a PI simulation, $\mathbf{b} \mathrm{MH}$ simulation, and $\mathbf{c}$ the change from the PI to the $\mathrm{MH}$ ( $\mathrm{MH}$ minus PI). The stippling in $\mathbf{c}$ indicates the difference of SSTA Stddev between PI and MH simulations is significant at $99 \%$ significance level, based on the $F$-test

FGOALS-g2 is composed of four component models, i.e., atmosphere, ocean, sea ice, and land surface, along with a central flux coupler. The oceanic component model is LICOM2.0 (Liu et al. 2012), which has $1^{\circ}$ horizontal resolution in the zonal direction and a variable grid in the meridional direction with $0.5^{\circ}$ resolution between $10^{\circ} \mathrm{S}$ and $10^{\circ} \mathrm{N}$, changing gradually to 
Table 1 Standard deviation of SSTA (units: K) in Niño3 and Niño3.4 regions, derived from the PI and $\mathrm{MH}$ simulations, and the change from the PI simulation to the $\mathrm{MH}$ simulation, together with the change rate of (MH minus PI)/PI

\begin{tabular}{lrr}
\hline & \multicolumn{1}{c}{ Niño3 } & Niño3.4 \\
\hline PI & 0.825 & 0.755 \\
MH & 0.591 & 0.536 \\
Change & -0.234 & -0.218 \\
Change rate & $-28.4 \%$ & $-29.0 \%$ \\
\hline
\end{tabular}

$1^{\circ}$ at about $20^{\circ} \mathrm{S}\left(20^{\circ} \mathrm{N}\right)$ and remaining at $1^{\circ}$ poleward. Vertically it has 30 levels with $10-\mathrm{m}$ resolution in the upper $150 \mathrm{~m}$. The atmospheric component model is the GAMIL version 2 (Li et al. 2013b), which has $26 \sigma$-levels. For more details about the major physical schemes in the oceanic and atmospheric components, one may refer to Liu et al. (2012) and Li et al. (2013b), respectively. The land component of FGOALS-g2 is the NCAR-CLM version 3 (Oleson et al. 2004), and the sea-ice component is the CICE4.0 (Hunke and Lipscomb 2008). The component models are coupled by version 6 of the NCAR flux coupler, in which the fluxes are exchanged at the interfaces of various component models with no flux correction. For details of the FGOALS-g2, please refer to Li et al. (2013a), Zheng and Yu (2013) and Zhou et al. (2014).

Based on the experiment protocols of PMIP3 and CMIP5, two suites of experiments by FGOALS-g2 were investigated in the present study. The first is the pre-industry simulation (hereafter PI), which represents the present-day climate, and the second is the MH simulation. The major difference of the forcing and boundary conditions between the two simulations lies in the difference in the orbital parameters. Specifically, the eccentricity changed from 0.0167724 in the PI to 0.018682 in the $\mathrm{MH}$, the obliquity changed from $23.446^{\circ}$ in the PI to $24.105^{\circ}$ in the $\mathrm{MH}$, and the angular precession changed from $102.04^{\circ}$ in the PI to $0.87^{\circ}$ in the MH. Additionally, the atmospheric methane concentration was reduced from the PI level of 760 to $650 \mathrm{ppb}$ for the MH. All the changes in orbital parameters induced insolation change and hence basic state change in the MH compared to the PI, e.g., an enhanced seasonal cycle in the Northern Hemisphere and a reduced seasonal cycle in the Southern Hemisphere. For more details about the experimental design and the basic simulation characteristics of the PI and MH simulations by FGOALS-g2, please refer to Zheng and Yu (2013). Both PI and MH simulations were integrated for more than 800 years, and the outputs of the last 100 years were used for comparison analysis in the present study.

\subsection{The BJ index}

The BJ index proposed by Jin et al. (2006) has been employed in some recent studies for ENSO stability analysis
(Kim and Jin 2011a, b; Jin 2014a, b; Liu et al. 2014; Chen et al. 2016; Hua et al. 2018; Wang et al. 2018). The method allows us to assess the contribution of different terms to the growth rate of a coupled ocean-atmosphere instability. In the present study, we employ the BJ index formulation in Kim and Jin (2011a, b), which is based on the framework of Jin et al. (2006) but contains several improvements. The formulation is listed as follows:

$\mathrm{BJ}=\frac{R-\varepsilon}{2}$

$$
\begin{aligned}
R= & -\left(a_{1} \frac{\langle\Delta \bar{u}\rangle_{E}}{L_{x}}+a_{2} \frac{\langle\Delta \bar{v}\rangle_{E}}{L_{y}}\right)-\alpha_{s}+\mu_{a} \beta_{u}\left\langle-\frac{\partial \bar{T}}{\partial x}\right\rangle_{E} \\
& +\mu_{a} \beta_{w}\left\langle-\frac{\partial \bar{T}}{\partial z}\right\rangle_{E}+\mu_{a} \beta_{h} a_{h}\left\langle\frac{\bar{w}}{H_{1}}\right\rangle_{E}
\end{aligned}
$$

$\frac{\partial\langle h\rangle_{w}}{\partial t}=-\varepsilon\langle h\rangle_{w}-\tilde{F}\left[\tau_{x}\right]$

$\langle\mathrm{Q}\rangle_{E}=-\alpha_{s}\langle T\rangle_{E}$

$\left\langle\mathrm{H}(\bar{w}) T_{\text {sub }}\right\rangle_{E}=a_{h}\langle h\rangle_{E}$

$\langle h\rangle_{E}-\langle h\rangle_{W}=\beta_{h}\left[\tau_{x}\right]$

$\langle\mathrm{H}(\bar{w}) w\rangle_{E}=\beta_{w}\left[\tau_{x}\right]$

$\langle\mathrm{u}\rangle_{E}=\beta_{u}\left[\tau_{x}\right]+\beta_{u h}\langle h\rangle_{w}$

$\left[\tau_{x}\right]=\mu_{a}\langle T\rangle_{E}$

Equation (1) elucidates the BJ index, which measures the growth rate discussed in this study. Equation (2) gives the corresponding dynamic and thermodynamic feedbacks. Equation (3) shows the heat content recharge/discharge process. In particular, the overbar denotes a climatological annual mean. $u, v$, and $w$ denote the zonal, meridional, and vertical oceanic current anomalies, respectively, and $T$ represents ocean temperature anomaly. The anomaly fields, such as $u, v, w$, and $T$, are obtained through removing their corresponding climatological seasonal cycle. \langle\rangle$_{E}$ and \langle\rangle$_{w}$ denote volume average quantities in the eastern and western boxes, respectively, from the surface to the base of the mixed layer. $L_{x}$ and $L_{y}$ are the longitudinal and latitudinal lengths of the eastern box, respectively. $a_{1}$ and $a_{2}$ are obtained using anomalous SST averaged zonally or meridionally at the boundaries of an area-averaged box and area-averaged SST anomaly (SSTA) in the eastern box. $H_{1}$ denotes a constant ocean mixed layer depth of $50 \mathrm{~m}$. $\mathrm{H}(\bar{w})=\left\{\begin{array}{l}1, \bar{w}>0 \\ 0, \bar{w} \leqslant 0\end{array}\right.$ is the step function to make sure only 
upward vertical motion is taken into account. $R$ represents the five feedbacks, which will be described in detail below. $\Delta$ in formula (2) indicates the ocean current differences between two boundaries. In formula (3), the first term on the right-hand side demonstrates the ocean adjustment characterized by a damping process at the rate of $\varepsilon$, and the second term denotes the Sverdrup transport across the equatorial Pacific basin. From formula (4) to formula (9), $\alpha_{s}$ denotes the response of the thermodynamic damping to SSTA; here, the thermodynamic damping is represented by the net surface heat flux anomaly divided by $\left(\rho C_{p} H_{1}\right), \rho$ denotes the density of seawater and $C_{p}$ is the specific heat capacity. $\mu_{a}$ denotes the response of zonal wind stress anomaly $\left(\tau_{x}^{\prime}\right)$ to SSTA; $\beta_{u}$ denotes the response of anomalous upper-ocean zonal current to $\tau_{x}^{\prime} ; \beta_{h}$ represents anomalous zonal slope of the equatorial thermocline adjusting to $\tau_{x}^{\prime} ; a_{h}$ indicates the effect of thermocline depth change on ocean subsurface temperature anomaly; and $\beta_{w}$ shows the response of ocean upwelling to wind forcing. [] in the BJ index indicates an anomaly averaged across the entire equatorial Pacific basin. More detailed description of the BJ index can be found in Kim and Jin (2011a, b).

Based on the above formulation, the main contributing terms of BJ index are discussed in the present study. They include two negative feedbacks, which are the dynamic damping by the mean advection (hereafter MA; $\left.-\left(a_{1} \frac{\langle\Delta \bar{u}\rangle_{E}}{L_{x}}+a_{2} \frac{\langle\Delta \bar{v}\rangle_{E}}{L_{y}}\right)\right)$ and the thermodynamic feedback (hereafter TD; $-\alpha_{s}$ ), and three positive feedbacks, which are the zonal advection feedback (hereafter ZA; $\mu_{a} \beta_{u}\left\langle-\frac{\partial \bar{T}}{\partial x}\right\rangle_{E}$ ), Ekman feedback (hereafter EK; $\mu_{a} \beta_{w}\left\langle-\frac{\partial \bar{T}}{\partial z}\right\rangle_{E}$ ), and thermocline feedback (hereafter $\mathrm{TH} ; \mu_{a} \beta_{h} a_{h}\left\langle\frac{\bar{w}}{H}\right\rangle_{E}$ ). The negative feedbacks damp the SST perturbation's growth, while the positive feedbacks favor the growth.

In this study, we chose the broad eastern box $\left(180^{\circ}-80^{\circ} \mathrm{W}, 5^{\circ} \mathrm{S}-5^{\circ} \mathrm{N}\right)$ when calculating the $\mathrm{BJ}$ index, following Chen et al. (2016) and Lüebbecke and McPhaden (2014). Note that the main conclusion and subsequent analysis in this study are not sensitive to a slight change in longitudinal boundaries of the box.

\section{Changes in the ENSO intensity and the BJ index}

\subsection{Changes in ENSO intensity in the MH compared to that in the PI}

Figure 1 shows the maps of SSTA standard deviation (Stddev) from the two simulations, and of the difference between the two ( $\mathrm{MH}$ simulation minus PI simulation). Here, the Stddev of SSTA is used as a metric to indicate the ENSO intensity. As clearly shown in Fig. 1c, the interannual variability of SSTA in the central and eastern equatorial Pacific was significantly weaker in the $\mathrm{MH}$ than in the PI, based on the $F$-test. The ENSO intensity was reduced by approximately $28 \%$ in the $\mathrm{MH}$ simulation compared to the modern-day climate simulation (Table 1), no matter whether the Niño3 region or Niño3.4 region is taken into account. As mentioned in the introduction, the CGCMs used in some other modeling studies (e.g., Zheng et al. 2008; An and Choi 2014) showed a relatively smaller decrease in ENSO intensity or even some marginal increase in ENSO intensity in the $\mathrm{MH}$ compared to that in the PI. In contrast, the CGCM of FGOALS-g2 used here presents a relatively marked decrease in the ENSO intensity, showing a better agreement with the ranges indicated by the paleo-proxies. This lends the confidence of using FGOALS-g2 for further in-depth analysis.

\subsection{Changes in the BJ index, and associated dynamic and thermodynamic processes}

To explore the physical processes responsible for the reduced ENSO variability in the $\mathrm{MH}$ compared to that in the $\mathrm{PI}$, we calculated the BJ index and the five main contributing terms to the BJ index for both simulations. As shown in the left-most bars in Fig. 2, the BJ index values derived from both simulations are negative, corresponding to a damped coupled ocean-atmosphere system in both PI and $\mathrm{MH}$. We further found that the value of the $\mathrm{BJ}$ index is more negative in the $\mathrm{MH}$ than in the PI. Based on the physical meaning

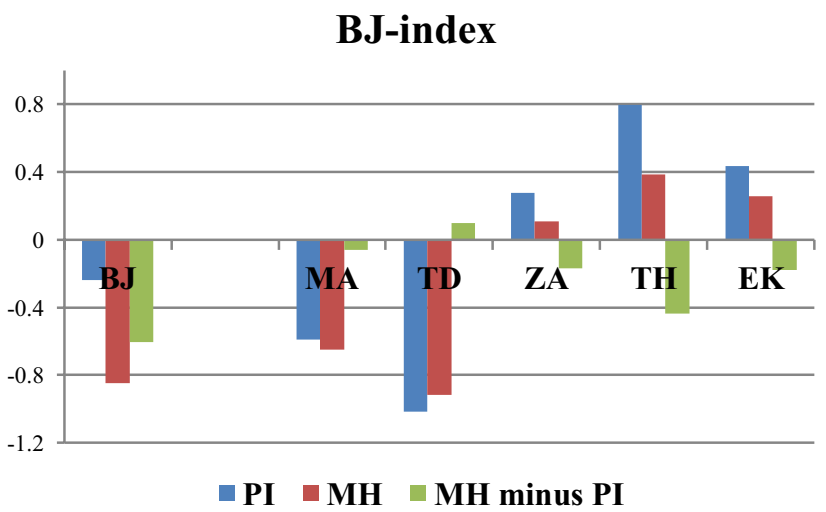

Fig. 2 The value of BJ index and its five contributing terms (units: year $^{-1}$ ), namely, mean advection feedback (MA), thermodynamic damping feedback (TD), zonal-advection feedback (ZA), thermocline feedback (TH), and Ekman feedback (EK), from the PI simulation (blue bars), the MH simulation (red bars), and their difference ( $\mathrm{MH}$ minus PI; green bars) 
of the $\mathrm{BJ}$ index, the fact that the $\mathrm{BJ}$ index exhibited a more negative value in the $\mathrm{MH}$ simulation indicates that the coupled ocean-atmosphere system was more stabilized in the MH. This more stabilized coupled ocean-atmosphere system was unfavorable for ENSO's growth rate, and thus caused a weakening in ENSO intensity in the $\mathrm{MH}$ compared to that in the PI. So, what are physical processes responsible for the more stabilized coupled system (or more negative BJ index) in the MH than in the PI? As depicted by the green bars in Fig. 2, among the five main contributing terms to the BJ index, the more negative $\mathrm{BJ}$ index in the $\mathrm{MH}$ primarily came from the weakening in the oceanic dynamic terms (including the TH term, ZA term, and EK term). We also noted that the $\mathrm{TD}$ term had a negative contribution to the $\mathrm{BJ}$ index change and ENSO intensity change. This indicates that the oceanic dynamic terms play an essential role in altering the $\mathrm{BJ}$ index and modulating the ENSO intensity in the MH, while the TD term works just to offset the ocean dynamic terms' effect. Therefore, we will mainly focus on the driving mechanisms for the weakened the ocean dynamic terms in the MH compared to those in the PI in the next section.

\section{Causes of the weakened oceanic dynamic terms in the $\mathrm{MH}$}

As analyzed above, the weaker ENSO intensity in the MH than in the PI is attributed to the reduction of the three oceanic dynamic terms (TH, ZA, and EK) in the MH. In this section, we investigate the causes of the changes in these three terms between PI and MH simulations. Note that each oceanic dynamic term involves two parts: one part is about the mean state, and the other part is about the air-sea feedback sub-processes, both of which might undergo changes to different extents in the MH compared to those in the PI. Thus, our strategy is to first probe the relative contribution by each factor to the change in a certain oceanic dynamic term. After identifying the dominant contributing factor, we will then investigate what causes the change in that factor during the $\mathrm{MH}$ compared to that during the PI.

\subsection{The TH feedback}

As described in Sect. 2.2, the TH term is written as: $T H=\mu_{a} * \beta_{h} * a_{h} *<\frac{\bar{W}}{H_{1}}>_{E}$. The TH term is usually called the thermocline feedback. It is associated with three air-sea feedback sub-processes (say, $\mu_{a}, \beta_{h}$, and $a_{h}$ ) and with mean upwelling $(\bar{W})$. The physical meaning of the thermocline feedback could simply be explained as follows. Given a perturbation of warm SSTA occurring in the eastern equatorial Pacific (EEP), this warm SSTA would lead to eastward zonal wind stress anomaly $\left(\tau_{x}^{\prime}\right)$. Here, the response of $\tau_{x}^{\prime}$ to SSTA is denoted by $\mu_{a}$. Then, an eastward $\tau_{x}^{\prime}$ would lead to thermocline shoaling in the western equatorial Pacific and thermocline deepening in the EEP. Here, the response of thermocline depth change to $\tau_{x}^{\prime}$ is represented by $\beta_{h}$. Then, the deepening of thermocline in the EEP would induce warmerthan-normal ocean subsurface temperature. The response of subsurface temperature change to thermocline change is represented by $a_{h}$. Last, considering the mean upwelling $(\bar{W})$ in the EEP, the warmer-than-normal subsurface temperature would, in turn, feed back to the mixed layer temperature and reinforce the warm SSTA in the EEP. Such a loop generates a positive thermocline feedback (i.e., the TH term), which is favorable for ENSO's growth.

The strengths of the air-sea feedback sub-processes $\left(\mu_{a}\right.$, $\beta_{h}$, and $a_{h}$ ) associated with the TH term are shown in Table 2 . Note that the strengths of $\beta_{h}$ and $a_{h}$ were reduced in the MH compared to those in the PI, while the strength of $\mu_{a}$ marginally decreased in the MH. Additionally, a slight difference in the mean upwelling between PI and MH simulations can be found (figure not shown). Naturally, one may ask which change played a dominant role in determining the TH term change, considering both the mean state part and the three air-sea feedback sub-processes would change in the $\mathrm{MH}$
Table 2 Regression coefficients associated with the BJ index derived from the PI and $\mathrm{MH}$ simulations, and their difference (MH minus PI)

\begin{tabular}{lcccccc}
\hline & $\alpha_{s}$, units: $\mathrm{s}^{-1}$ & $\mu_{a},\left(\mathrm{~N} \mathrm{~m}^{-2}\right) / \mathrm{K}$ & $\begin{array}{l}\beta_{u},\left(\mathrm{~m} \mathrm{~s}^{-1}\right) / \\
\left(\mathrm{N} \mathrm{m}^{-2}\right)\end{array}$ & $\beta_{w},\left(\mathrm{~m} \mathrm{~s}^{-1}\right) /\left(\mathrm{N} \mathrm{m}^{-2}\right)$ & $\beta_{h}, \mathrm{~m} /\left(\mathrm{N} \mathrm{m}^{-2}\right)$ & $a_{h}, \mathrm{~K} / \mathrm{m}$ \\
\hline PI & $-6.44 \times 10^{-8}$ & $3.7 \times 10^{-3}$ & 7.5 & $-1.50 \times 10^{-4}$ & 5.6 & 29.6 \\
MH & $-5.82 \times 10^{-8}$ & $3.6 \times 10^{-3}$ & 4.0 & $-0.97 \times 10^{-4}$ & 2.9 & 26.3 \\
Change & $0.62 \times 10^{-8}$ & $-0.1 \times 10^{-3}$ & -3.6 & $0.53 \times 10^{-4}$ & -2.7 & -3.3 \\
(MH- & & & & & & \\
PI) & & & & & & \\
\hline
\end{tabular}

$\alpha_{s}$ denotes the response of thermodynamic damping (here the thermodynamic damping was represented by the net surface heat flux anomaly divided by $\left(\rho C_{p} H_{1}\right)$, and the positive value means ocean receives heat) to sea surface temperature anomalies (SSTAs). $\mu_{a}$ denotes the response of zonal wind stress anomaly $\left(\tau_{x}^{\prime}\right)$ to SSTA; $\beta_{u}$ denotes the response of anomalous oceanic zonal current $\left(u^{\prime}\right)$ to $\tau_{x}^{\prime} ; \beta_{w}$ denotes the response of anomalous oceanic vertical current $\left(w^{\prime}\right)$ to $\tau_{x}^{\prime} ; \beta_{h}$ represents anomalous zonal slope of the equatorial thermocline adjusting to $\tau_{x}^{\prime}$; and $a_{h}$ indicates the effect of thermocline depth change on ocean subsurface temperature anomaly 


\section{Relative contribution to $T H$ change}

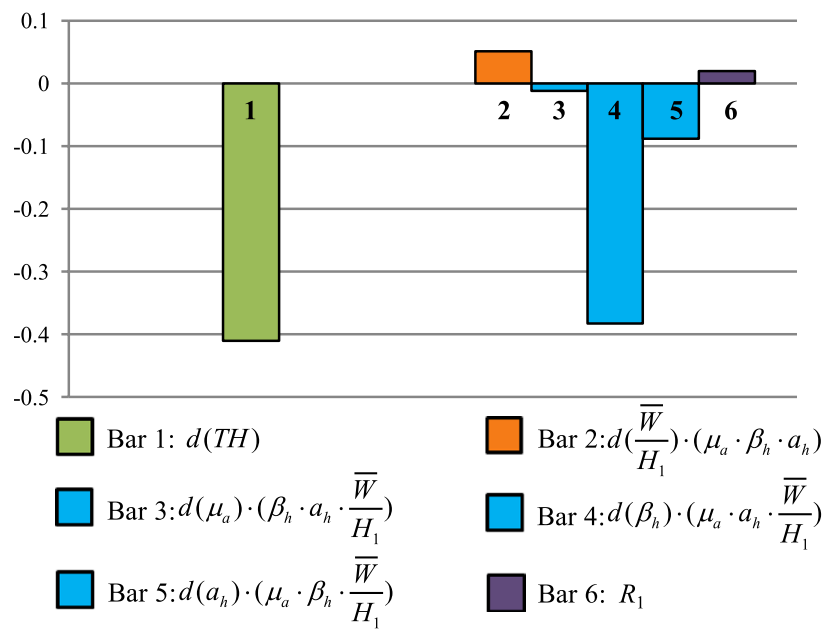

Fig. 3 Green bar denotes the change of TH term [bar 1: $d(T H)]$. Note that $d()$ represents the difference between PI and MH simulations (MH minus PI). Orange bar [bar2: $\left.d\left(\frac{\bar{W}}{H_{1}}\right) \cdot\left(\mu_{a} \cdot \beta_{h} \cdot a_{h}\right)\right]$ indicates the contribution from the mean state part change $\left[d\left(\frac{\bar{W}}{H_{1}}\right)\right]$ to the change of TH term. Three blue bars, respectively, represent the contributions from the changes of air-sea feedbacks to the change of TH term. Specifically, bar $3\left[d\left(\mu_{a}\right) \cdot\left(\beta_{h} \cdot a_{h} \cdot \frac{\bar{W}}{H_{1}}\right)\right]$ indicates the contribution from $d\left(\mu_{a}\right)$ to the change of TH term, bar $4\left[d\left(\beta_{h}\right) \cdot\left(\mu_{a} \cdot a_{h} \cdot \frac{\bar{W}}{H_{1}}\right)\right]$ indicates the contribution from $d\left(\beta_{h}\right)$ to the change of TH term, and bar 5 $\left[d\left(a_{h}\right) \cdot\left(\mu_{a} \cdot \beta_{h} \cdot \frac{\bar{W}}{H_{1}}\right)\right]$ indicates the contribution from $d\left(a_{h}\right)$ to the change of TH term. $R_{I}$ is the residual, which indicates the contribution from the covariant changes of both the mean state part and the air-sea feedbacks. Units: year ${ }^{-1}$

compared to those in the PI. Based on the total derivative relationship (for details, please refer to the equations in the "Appendix"), the contribution to the change in the TH term was decomposed into three parts: the contribution due to the change in the mean state, the contribution due to the change in the air-sea feedbacks, and the contribution due to the covariant changes in both mean state and the air-sea feedbacks.

As shown in Fig. 3, the weakened TH term in the MH (see bar 1 in Fig. 3) was primarily caused by the change in $\beta_{h}$ (bar 4 in Fig. 3), and the contribution due to the change in $a_{h}$ played a secondary role (bar 5 in Fig. 3). In contrast, the change in $\mu_{a}$ (bar 3 in Fig. 3 ) had less contribution to the weakened TH term, and both the contribution due to the change in mean upwelling (bar 2 in Fig. 3) and that due to the covariant changes of the mean state and the air-sea feedbacks (bar 6 in Fig. 3) were negative. Thus, it is concluded that the changes in $\beta_{h}$ and $a_{h}$ (especially the change in $\beta_{h}$ ) were the dominant contributing factors for causing the weakened TH term in the MH.

To investigate the cause of the change in $\beta_{h}$, we first plotted the map of the response of sea surface height anomaly (a) PI

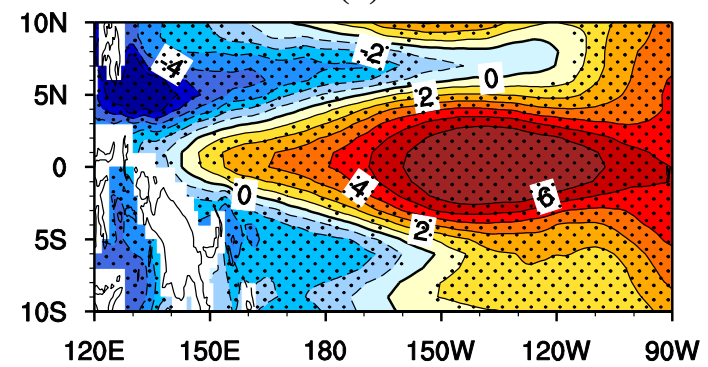

(b) $\mathrm{MH}$

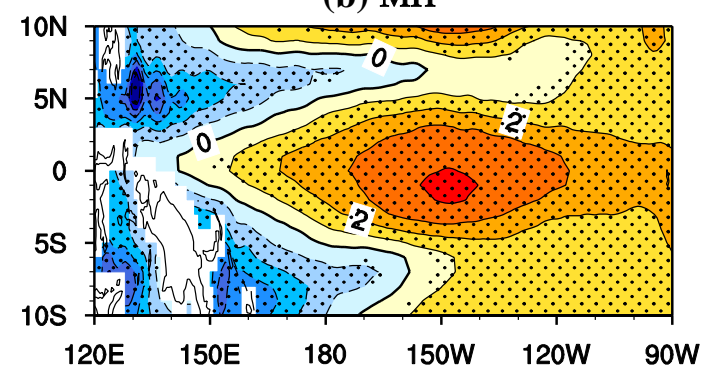

(c) Change

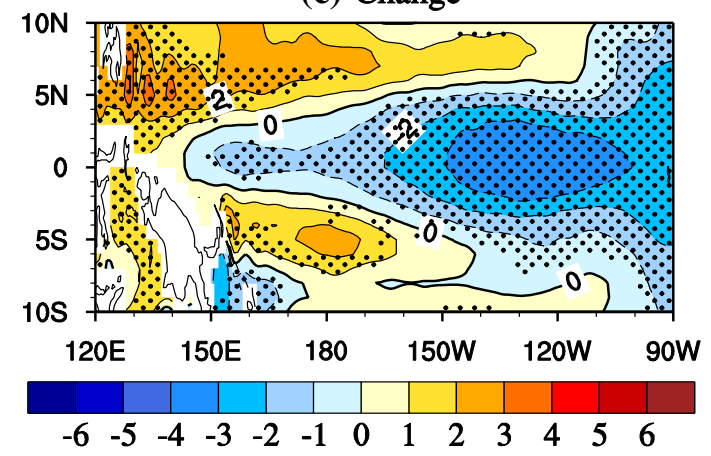

Fig. 4 The horizontal map of the response of thermocline depth anomaly $\left(D^{\prime}\right)$ to zonal wind stress anomaly $\left[\beta_{h}\right.$; units: $\left.\mathrm{m}\left(\mathrm{N} \mathrm{m}^{-2}\right)^{-1}\right]$, derived from a PI simulation, b MH simulation, and $\mathbf{c}$ their difference. Here, sea surface height anomaly $\left(\mathrm{SSH}^{\prime}\right)$ is used as a proxy of $D^{\prime}$, based on the linear $S S H^{\prime}-D^{\prime}$ relationship. The $D^{\prime}$ response to zonal wind stress anomaly is obtained through regressing the $S S H^{\prime}$ field onto the equatorial Pacific zonal wind stress anomaly averaged over $120^{\circ} \mathrm{E}-80^{\circ} \mathrm{W}, 5^{\circ} \mathrm{S}-5^{\circ} \mathrm{N}$. The stippling in top and middle panels indicates the regression coefficient exceeding a confidence level of $99 \%$ using Student's $t$ test. The stippling in the bottom panels indicates the changes in the regressions coefficients exceeding a confidence level of $95 \%$ using a $Z$ test

$\left(S S H^{\prime}\right.$, a proxy of the thermocline depth anomaly [ $\left.\left.D^{\prime}\right]\right)$ to $\tau_{x}^{\prime}$, derived from PI simulation, $\mathrm{MH}$ simulation, and their difference (Fig. 4a-c). Note that in both PI and MH simulations (Fig. 4a,b), in response to a unit eastward $\tau_{x}^{\prime}$ in the equatorial Pacific, the tilt of the thermocline would be less, accompanied with a deepening of the thermocline depth (i.e., positive $D^{\prime}$ ) in the EEP. Although such characteristics of $\beta_{h}$ were captured by both PI and MH simulations (Fig. 4a, b), the difference map shows that the strength of $D^{\prime}$ response to $\tau_{x}^{\prime}$ was indeed weakened in the MH compared to that in the 


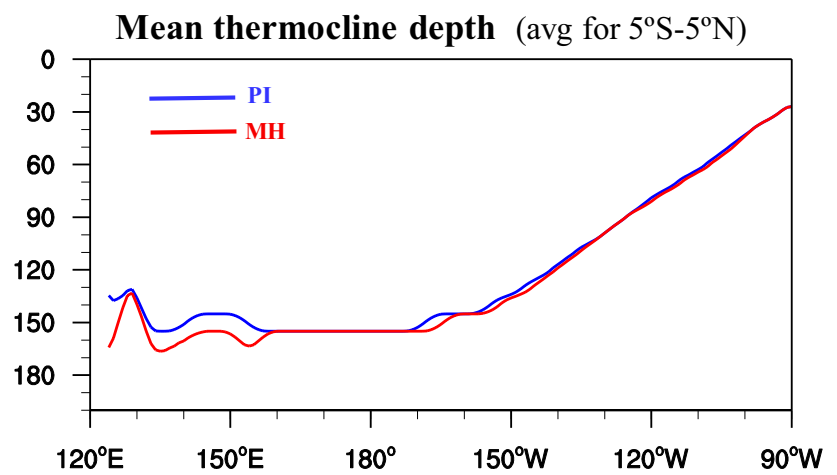

Fig. 5 Climatological mean thermocline depth (units: m) along the equator (averaged between $5^{\circ} \mathrm{S}$ and $5^{\circ} \mathrm{N}$ ), derived from the PI simulation (blue curve) and $\mathrm{MH}$ simulation (red curve). Here, the depth of mean thermocline is estimated by using the depth where the maximum vertical gradient of mean temperature is located

PI (Fig. 4c). Such contrasting $D^{\prime}$ response between PI and $\mathrm{MH}$ simulations is consistent with the contrasting values of $\beta_{h}$ in Table 2.

Why did the $S S H^{\prime}$ or $D^{\prime}$ response to $\tau_{x}^{\prime}$ become weakened during the MH compared to that during the PI? According to the Sverdrup balance relationship (Jin 1997; Li 1997), we may obtain $\frac{\partial D^{\prime}}{\partial x}=\frac{\tau_{x}^{\prime}}{\rho_{0} g \bar{H}}$, where $D^{\prime}$ and $\bar{H}$ are anomalous and mean thermocline depths, respectively; $\rho_{0}$ is seawater density; and $g$ is the reduced gravity. We first examine whether there is difference in the mean equatorial thermocline depth between PI and MH simulations. We find there is negligible difference in the mean thermocline depth along the equator between PI and MH simulations (Fig. 5). So, we further

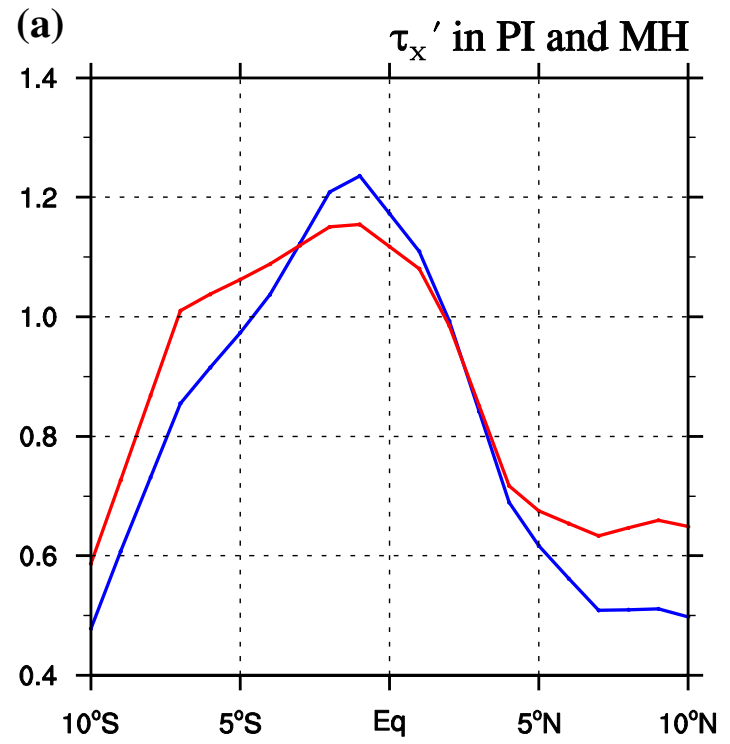

Fig. 6 Meridional structure of normalized $\tau_{x}^{\prime}$ field [units: $\mathrm{N} \mathrm{m}^{-2}(\mathrm{~N}$ $\left.\mathrm{m}^{-2}\right)^{-1}$ ] averaged over the Niño4 longitude range $\left(160^{\circ} \mathrm{E}-150^{\circ} \mathrm{W}\right)$, derived from a PI simulation (blue) and $\mathrm{MH}$ simulation (red), and $\mathbf{b}$ examine whether there exists any difference in the meridional structure of $\tau_{x}^{\prime}$, because it is suggested that in the same CGCM, a change in the meridional structure of $\tau_{x}^{\prime}$ would induce a change in the $D^{\prime}$ response to $\tau_{x}^{\prime}$ (Su et al. 2014; Chen et al. 2015, 2017). Then we followed Chen et al. (2015) and plotted the corresponding meridional structures of $\tau_{x}^{\prime}$ derived from the PI and MH simulations. As depicted in Fig. 6, there is a pronounced change in the meridional structure of $\tau_{x}^{\prime}$ in the MH compared to that in the PI: the meridional structure of $\tau_{x}^{\prime}$ became wider and flatter in the MH compared to that in the PI. This indicates that the distribution of $\tau_{x}^{\prime}$ at the equator would be weaker in the MH compared to that in the PI, given the same box-averaged $\tau_{x}^{\prime}$ forcing in the Niño4 region. Accordingly, a weaker $\tau_{x}^{\prime}$ at the equator could induce a smaller $D^{\prime}$ response in the MH compared to that in the PI, based on the Sverdrup relationship. We also examined the meridional structure of SSTA derived from the two simulations. As presented in Fig. 7, we find the meridional structure of SSTA was also wider and flatter in the MH than that in the PI, which was in agreement with the change in meridional structure of $\tau_{x}^{\prime}$. It is no wonder that the meridional structures of ENSO-related fields (say, SSTA and $\tau_{x}^{\prime}$ ) underwent similar changes, because SSTA and $\tau_{x}^{\prime}$ are physically coupled.

Next we investigate the cause for the flatter ENSO meridional structure in the $\mathrm{MH}$ compared to that in the PI. Previous studies (Zhang et al. 2009, 2013; Zhang and Jin 2012) suggested that the ENSO meridional structure be regulated by the climatological meridional current or the so-called Pacific subtropical cell (STC; McCreary and Lu 1994). Figure 8 shows the climatological mean STC from the PI simulation

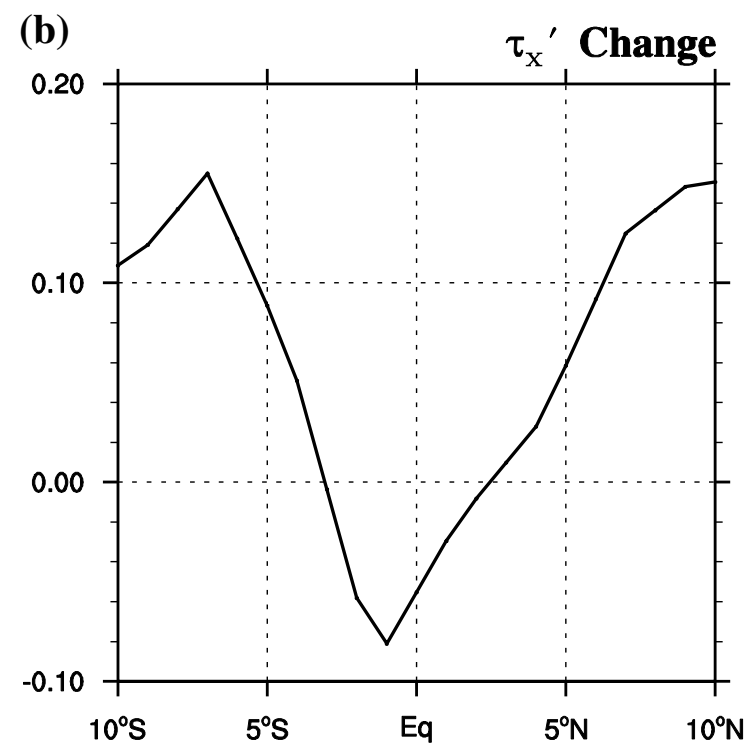

the difference between the PI and MH simulations (black). The normalized $\tau_{x}^{\prime}$ field was obtained through regressing the $\tau_{x}^{\prime}$ field onto the time series of Niño4-averaged $\tau_{x}^{\prime}$ 


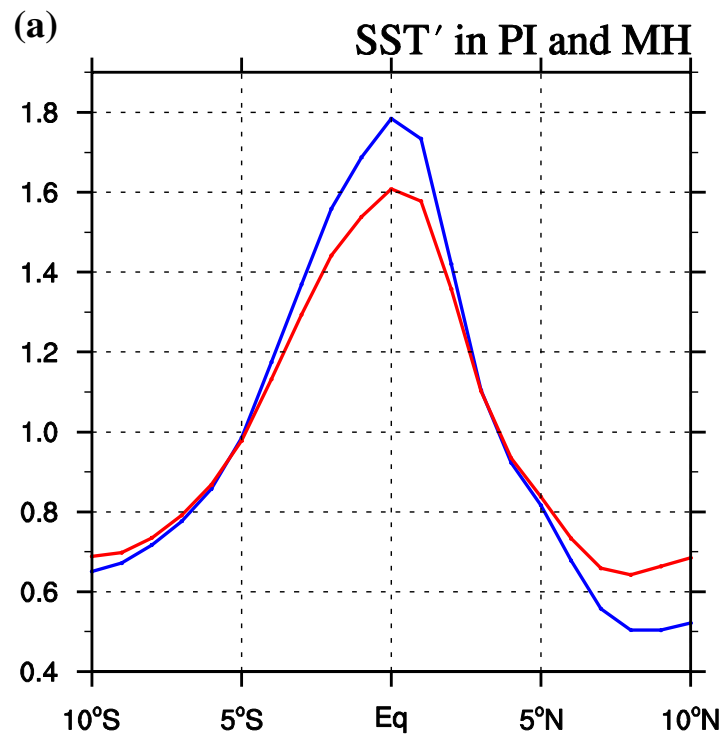

Fig. 7 Meridional structure of normalized SSTA-Stddev field (units: $\mathrm{K} / \mathrm{K})$ averaged over the eastern box longitude range $\left(180^{\circ}-80^{\circ} \mathrm{W}\right)$, derived from a PI simulation (blue) and MH simulation (red), and b the difference between PI and MH simulations (black). Here, the

(Fig. 8a), MH simulation (Fig. 8b), and their difference (Fig. 8c). The strength of mean STC is represented by the meridional overturning stream function (MOSF). The mean MOSFs in the PI and MH simulations (Fig. 8a, b) indicate a poleward transport of water mass in the surface layer, and then the meridional transport alters sign from poleward to equatorward below the maximum MOSF. The climatological STC became strengthened in the MH compared to that in the PI (Fig. 8c). This means the poleward transport of surfacelayer seawater became strengthened in the $\mathrm{MH}$ compared to that in the PI. As a result, the strengthened surface poleward transport led the ENSO-related anomaly fields (SSTA and $\tau_{x}^{\prime}$ ) to become more outspread relative to the equator, corresponding to a wider and flatter ENSO meridional structure in the $\mathrm{MH}$.

As described above, the strength of $a_{h}$ was slightly reduced in the $\mathrm{MH}$ than in the PI, and the reduced $a_{h}$ played a secondary role in leading to the reduction in the $\mathrm{TH}$ term during the $\mathrm{MH}$ compared to that during the PI (bar 5 in Fig. 3). Thus, we next investigate what caused the reduction in $a_{h}$ during the $\mathrm{MH}$ compared to that during the PI. Figure 9 shows the vertical distribution of mean temperature from PI and MH simulations. The vertical stratification of mean temperature was relatively diffused (sharper) in the MH (the PI). This indicates that the ocean subsurface temperature would change less (more) in the MH (the PI), given the same unit change of thermocline depth anomaly. It is worth mentioning that this viewpoint that the change in vertical stratification of mean temperature could alter the strength of $a_{h}$ and further affect the TH term and ENSO's

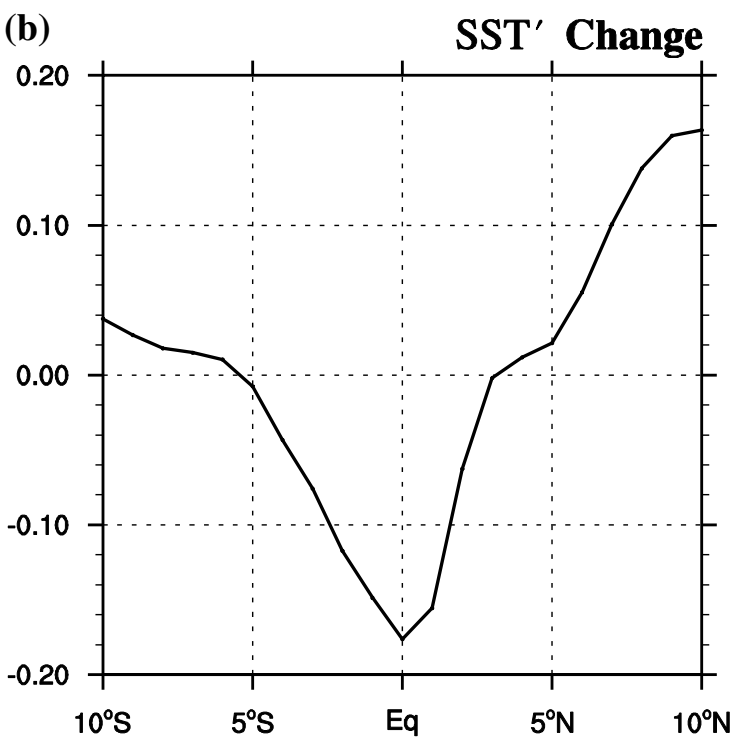

SSTA-Stddev field is normalized by the area-averaged value of SSTA-Stddev over the eastern Pacific region $\left(180^{\circ}-80^{\circ} \mathrm{W}, 10^{\circ} \mathrm{S}-\right.$ $\left.10^{\circ} \mathrm{N}\right)$

amplitude, also appeared in previous studies (e.g., Roberts et al. 2014); however, through quantitatively diagnosing the relative contribution from the change of each air-sea feedback to the TH term change, the current modeling study presented that the change in the $\mathrm{TH}$ term primarily arose from the change in $\beta_{h}$, and that the change in $a_{h}$ only played a secondary role. Additionally, their study only attributed the reduction in the oceanic temperature-wind feedback (i.e., the combination of $\beta_{h}$ and $a_{h}$ ) to the more diffused thermocline. However, through analyzing $a_{h}$ and $\beta_{h}$ individually, we suggested that the more diffused thermocline was primarily responsible for the reduced $a_{h}$, and the reduced $\beta_{h}$ was induced by the enhanced mean STC.

\subsection{The ZA feedback}

The ZA term is written as: $Z A=\mu_{a} * \beta_{u} *\left\langle-\frac{\partial \bar{T}}{\partial x}\right\rangle_{E}$. The ZA term is usually called the zonal advection feedback, which is associated with the zonal gradient of mean temperature $\left(\frac{\partial \bar{T}}{\partial x}\right)$ and two air-sea feedback sub-processes $\left(\mu_{a}\right.$ and $\left.\beta_{u}\right)$. Given both the mean state part and the air-sea feedback subprocesses would change in the MH compared to those in the PI, which one played a dominant role in determining the change in the ZA term? Based on the total derivative relationship [see Eq. (14) in the "Appendix"], we isolated the relative contributions from the change of the mean state part and the changes in the two air-sea feedbacks. As shown in Fig. 10, the weakened ZA term in the MH (see bar 1 in Fig. 10) was primarily caused by the change in $\beta_{u}$ (bar 4 in 
Meridional overturning stream function (Sv)
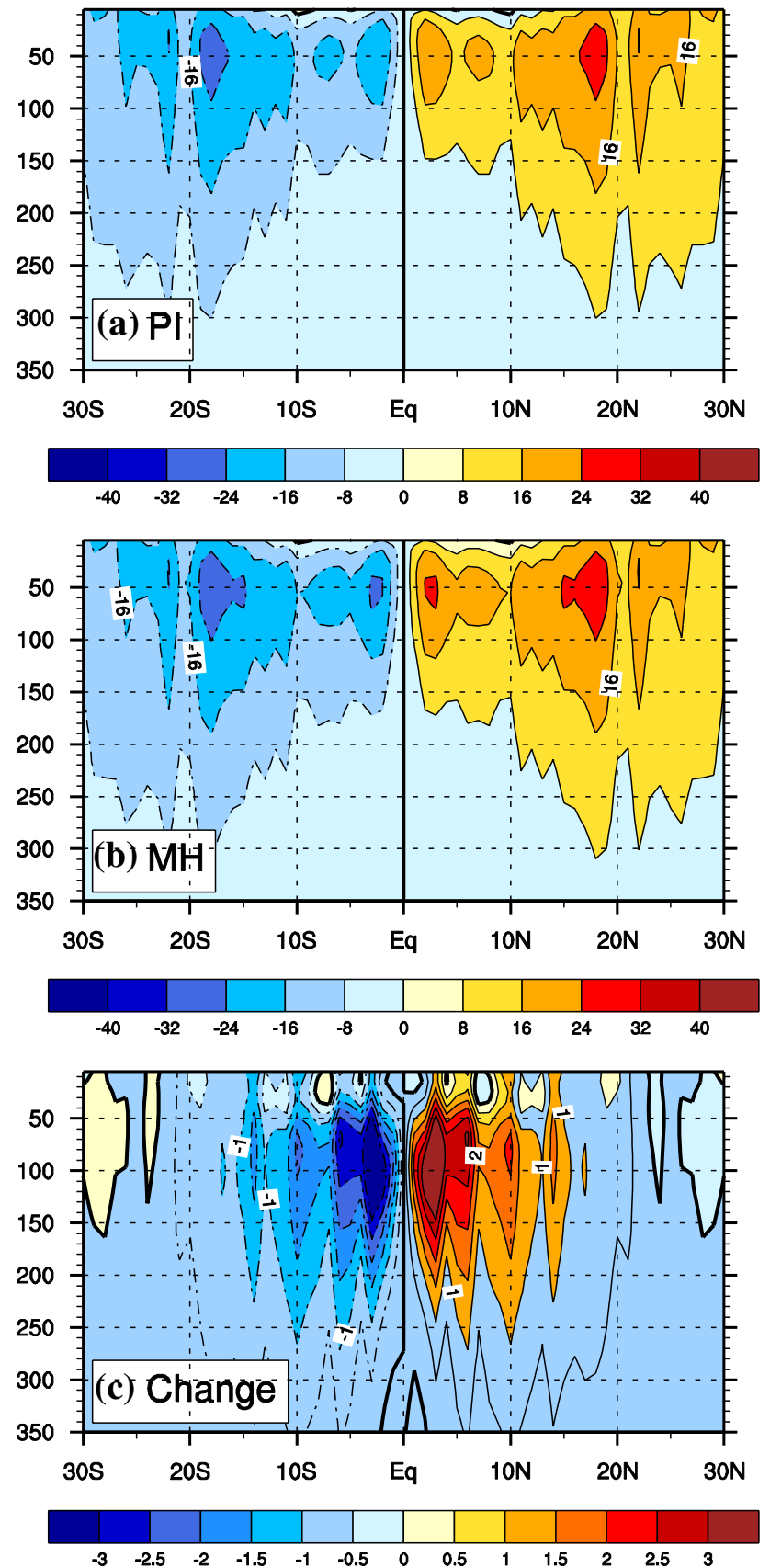

Fig. 8 Climatological mean subtropical cell (STC) derived from a PI simulation, b MH simulation, and $\mathbf{c}$ their difference (MH minus PI). The units for depth along the y axis are meters. Here, the STC is measured by the meridional overturning stream function (positive values indicate clockwise rotation; units: Sv), and only its antisymmetric component relative to the equator is shown

Fig. 10). In contrast, the contribution by the change in $\frac{\partial \bar{T}}{\partial x}$ (bar 2 in Fig. 10), the contribution by the change in $\mu_{a}$ (bar 3 in Fig. 10) and the contribution by the covariant changes of the mean state and the air-sea feedback (bar 5 in Fig. 10) were

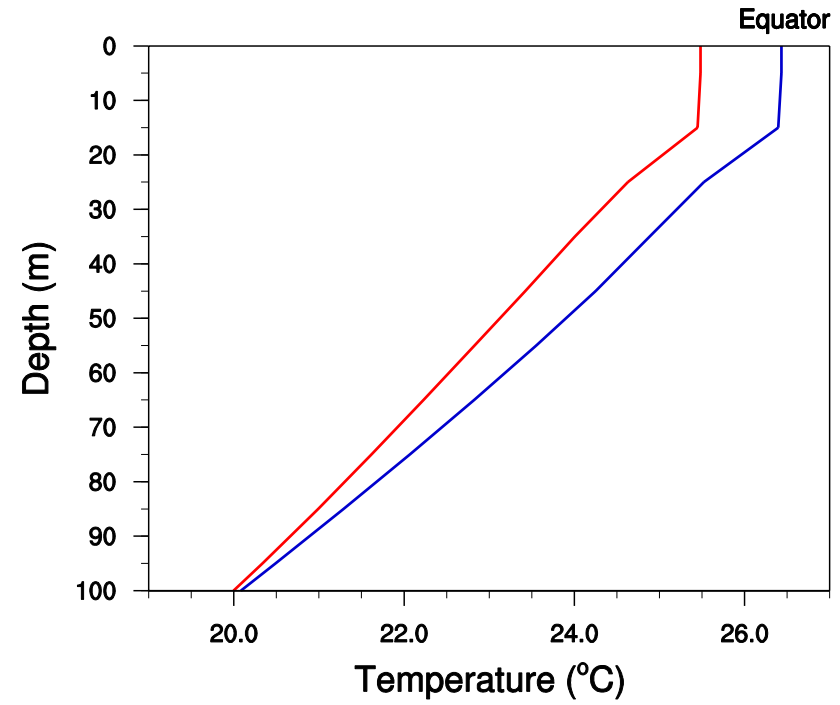

Fig. 9 Vertical distribution of mean temperature over the eastern box $\left(180^{\circ}-80^{\circ} \mathrm{W}, 5^{\circ} \mathrm{S}-5^{\circ} \mathrm{N}\right)$ from the PI simulation (blue) and $\mathrm{MH}$ simulation (red)

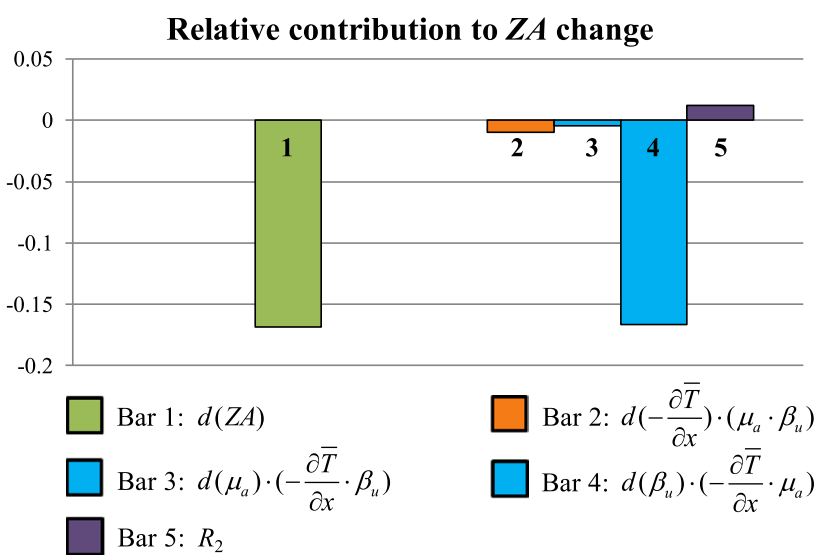

Fig. 10 Green bar denotes the change of ZA term [bar 1: $d(Z A)]$. Orange bar $\left[\operatorname{bar} 2: d\left(-\frac{\partial \bar{T}}{\partial x}\right) \cdot\left(\mu_{a} \cdot \beta_{u}\right)\right]$ indicates the contribution from the mean state part change $\left[d\left(-\frac{\partial \bar{T}}{\partial x}\right)\right]$ to the change of TH term. Two blue bars, respectively, represent the contributions from the changes of air-sea feedbacks to the change of ZA term. Specifically, bar 3 $\left[d\left(\mu_{a}\right) \cdot\left(-\frac{\partial \bar{T}}{\partial x} \cdot \beta_{u}\right)\right]$ indicates the contribution from $d\left(\mu_{a}\right)$ to the change of ZA term, and bar $4\left[d\left(\beta_{u}\right) \cdot\left(-\frac{\partial \bar{T}}{\partial x} \cdot \mu_{a}\right)\right]$ indicates the contribution from $d\left(\beta_{u}\right)$ to the change of ZA term. $R_{2}$ is the residual, which indicates the contribution from the covariant changes of both the mean state part and the air-sea feedbacks. Units: year $^{-1}$

all negligible. Thus, next we explore the cause of the change in $\beta_{u}$ during the $\mathrm{MH}$ compared to that during the PI.

$\beta_{u}$ represents the response of anomalous oceanic zonal current (hereafter $u^{\prime}$ ) to $\tau_{x}^{\prime}$. We first plot the vertical-zonal section of the response of $u^{\prime}$ to $\tau_{x}^{\prime}$ across the equatorial 
(a) $\mathrm{PI}$

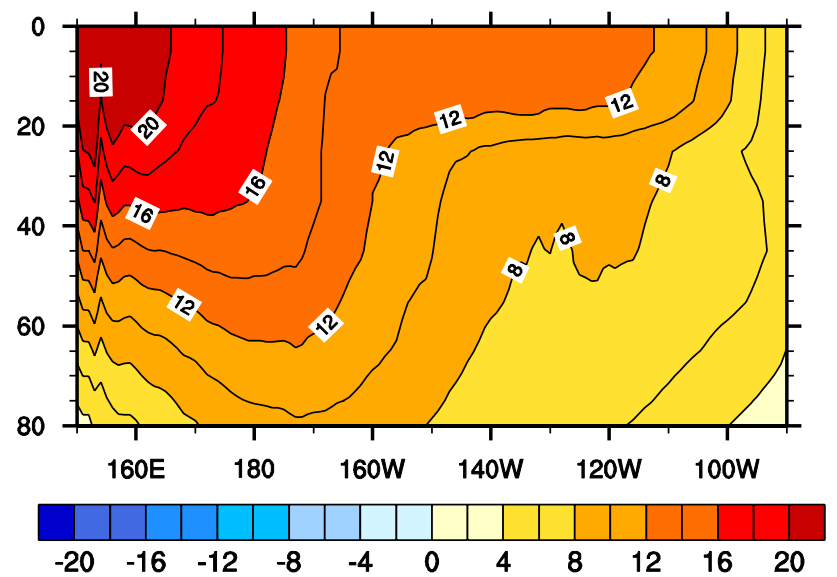

(b) $\mathrm{MH}$

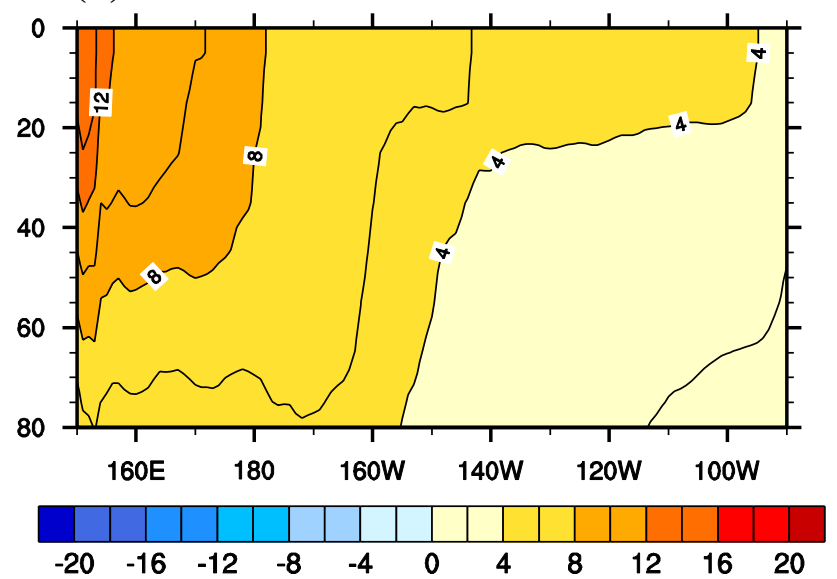

(c) $\mathrm{MH}$ minus $\mathrm{PI}$

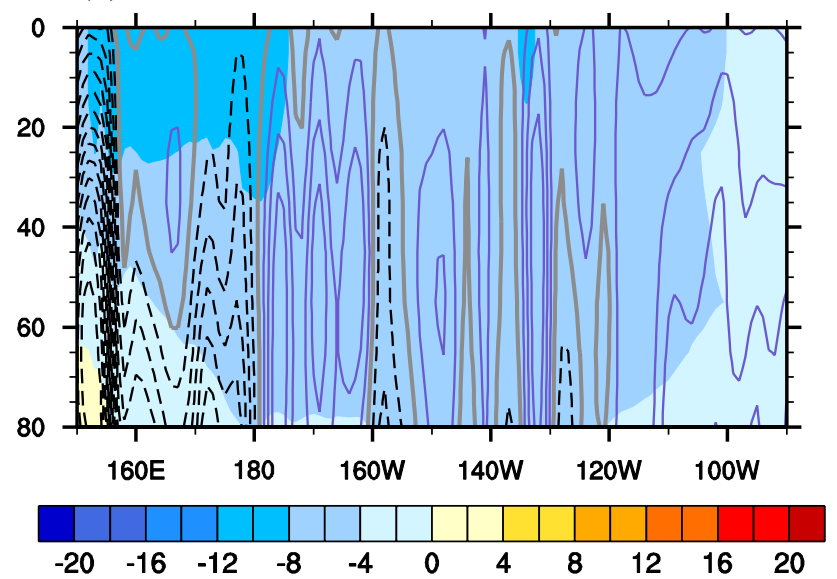

Fig. 11 a Vertical-zonal section along the equator $\left(5^{\circ} \mathrm{S}-5^{\circ} \mathrm{N}\right.$ averaged) of the response of $u^{\prime}$ to the equatorial Pacific $\tau_{x}^{\prime}$ [shading; units: $\left.\left(\mathrm{m} \mathrm{s}^{-1}\right) /\left(\mathrm{N} \mathrm{m}^{-2}\right)\right]$, derived from a PI simulation, b $\mathrm{MH}$ simulation, and $\mathbf{c}$ their difference ( $\mathrm{MH}$ minus PI). The contours in $\mathbf{c}$ denote the difference in the response of $w^{\prime}$ to the equatorial Pacific $\tau_{x}^{\prime}$ [contour interval is $1.5 \times 10^{-5}\left(\mathrm{~m} \mathrm{~s}^{-1}\right) /\left(\mathrm{N} \mathrm{m}^{-2}\right)$; gray curve denotes zero line; slate-blue solid curves denote positive values; and black dashed curves denote negative values]
Table 3 Responses of zonal current anomaly $\left(u^{\prime}\right)$ averaged over 0-50 m, zonal geostrophic current anomaly $\left(u_{\mathrm{g}}{ }^{\prime}\right)$, and zonal Ekman current anomaly $\left(u_{e}{ }^{\prime}\right)$ along the central-eastern equatorial Pacific $\left(180^{\circ}-80^{\circ} \mathrm{W}\right.$, $2^{\circ} \mathrm{S}-2^{\circ} \mathrm{N}$ ) to zonal wind stress anomaly derived from the PI and $\mathrm{MH}$ simulations, and their difference

Pacific (see the shading in Fig. 11a-c). As shown in Fig. 11a, $\mathrm{b}$, in response to a unit eastward $\tau_{x}^{\prime}$ in the equatorial Pacific, there would be eastward anomalous $u^{\prime}$ along the equator in both PI and MH simulations. Furthermore, the difference map shows that the strength of $\beta_{u}$ (i.e., $u^{\prime}$ response to $\tau_{x}^{\prime}$ ) was indeed weakened in the MH compared to that in the PI (see the shading in Fig. 11c). The weakening in $u^{\prime}$ response to $\tau_{x}^{\prime}$ shown in Fig. $11 \mathrm{c}$ is consistent with the reduced strength of $\beta_{u}$ in the MH compared to that in the PI (as shown in Table 2).

Why was $\beta_{u}$ weaker in the MH than in the PI? Based on previous studies (e.g., Su et al. 2010, 2014), $u^{\prime}$ could be decomposed into two parts: one is the anomalous zonal geostrophic current written as $u_{g}^{\prime}=-\frac{g \partial^{2} D^{\prime}}{\beta \partial y^{2}}$, where $g$ is the reduced gravity, $D^{\prime}$ is thermocline depth anomaly, and $\beta$ is planetary vorticity gradient; and the other is the anomalous Ekman current written as $u_{e}^{\prime}=\frac{1}{\rho H_{1}} \frac{r_{s} \tau_{x}^{\prime}+\beta y \tau_{y}^{\prime}}{r_{s}^{2}+(\beta y)^{2}}$, where $\tau_{y}^{\prime}$ is meridional wind stress anomaly, $H_{l}$ denotes mixed layer depth, and $r_{s}$ is a constant Rayleigh damping coefficient. As shown in Table 3, the diagnosed results reveal that in both simulations, the response of $u^{\prime}$ to $\tau_{x}^{\prime}$ was mainly determined by the response of $u_{g}{ }^{\prime}$ to $\tau_{x}^{\prime}$, while $u_{e}^{\prime}$ had less contribution. Previous studies (e.g., Su et al. 2014; Chen et al. 2015, 2017) suggested that $u_{g}{ }^{\prime}$ response to $\tau_{x}^{\prime}$ could be traced back to the $D^{\prime}$ response to $\tau_{x}^{\prime}$. The corresponding physical chain is simply explained below. Recalling the parabolic shape of the $D^{\prime}$ response (see Fig. 4a, b), the maximum $D^{\prime}$ was located on the equator with a decrease poleward, thus the stronger (weaker) $D^{\prime}$ response corresponded to a larger (smaller) value of $-\frac{\partial^{2} D^{\prime}}{\partial y^{2}}$ and a stronger (weaker) $u_{g}{ }^{\prime}$ response. This indicates that the difference of $\beta_{u}$ (i.e., $u^{\prime}$ response to $\tau_{x}^{\prime}$ ) between PI and MH simulations also resulted from the aforementioned difference in $D^{\prime}$ response to $\tau_{x}^{\prime}$. A simple flow chart may be used to illustrate the cause of the ZA term change from PI simulation to $\mathrm{MH}$ simulation, that is, $\mathrm{SSH}^{\prime}$ $\sim D^{\prime}-->u_{\mathrm{g}}{ }^{\prime}-->u^{\prime}-->\beta_{u^{--}}>\mathrm{ZA}$. 


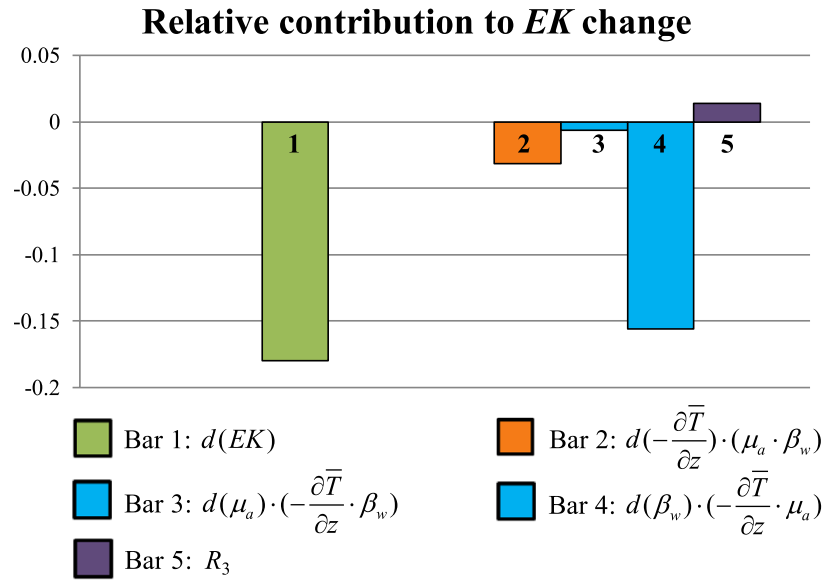

Fig. 12 Green bar denotes the change of EK term [bar 1: $d(E K)]$. Orange bar $\left[\operatorname{bar} 2: d\left(-\frac{\partial \bar{T}}{\partial z}\right) \cdot\left(\mu_{a} \cdot \beta_{w}\right)\right]$ indicates the contribution from the mean state part change $\left[d\left(-\frac{\partial \bar{T}}{\partial z}\right)\right]$ to the change of TH term. Two blue bars, respectively, represent the contributions from the changes of air-sea feedbacks to the change of EK term. Specifically, bar 3 $\left[d\left(\mu_{a}\right) \cdot\left(-\frac{\partial \bar{T}}{\partial z} \cdot \beta_{w}\right)\right]$ indicates the contribution from $d\left(\mu_{a}\right)$ to the change of ZA term, and bar $4\left[d\left(\beta_{w}\right) \cdot\left(-\frac{\partial \bar{T}}{\partial z} \cdot \mu_{a}\right)\right]$ indicates the contribution from $d\left(\beta_{w}\right)$ to the change of ZA term. $R_{3}$ denotes the residual, which indicates the contribution from the covariant changes of both the mean state part and the air-sea feedbacks. Units: year $^{-1}$

\subsection{The EK feedback}

The EK formulation is written as: $E K=\mu_{a} * \beta_{w} *\left\langle-\frac{\partial \bar{T}}{\partial z}\right\rangle_{E}$. The EK term is usually called the anomalous upwelling or Ekman feedback, which is associated with the vertical gradient of mean temperature $\left(\frac{\partial \bar{T}}{\partial z}\right)$ and two air-sea feedback subprocesses $\left(\mu_{a}\right.$ and $\left.\beta_{w}\right)$. Given both the mean state part and the air-sea feedbacks would change in the MH compared to that in the PI, it is necessary to first identify the relative contributions from the change of the mean state part and the changes in the two air-sea feedbacks. Based on the total derivative relationship [see Eq. (45) in the "Appendix"], we first quantitatively calculated the relative contributions from the change of the mean state and the changes in the two airsea feedbacks to the change in EK term. Our results show that the weakened EK term in the MH (see bar 1 in Fig. 12) was primarily caused by the change in $\beta_{w}$ (bar 4 in Fig. 12), followed by the change in $\frac{\partial \bar{T}}{\partial z}$ (bar 2 in Fig. 12). In contrast, the contribution by the change in $\mu_{a}$ (bar 3 in Fig. 12) and the contribution by the covariant changes of the mean state and the air-sea feedback (bar 5 in Fig. 12) were negligible.

Understanding the changes in $\frac{\partial T}{\partial z}$ and $\beta_{w}$ is straightforward. Recall that the vertical distribution of mean temperature along the equator (Fig. 9) was relatively more diffused (sharp) in the MH (the PI), corresponding to a relatively weaker (stronger) $\frac{\partial \bar{T}}{\partial z}$. As such, the reduced $\frac{\partial \bar{T}}{\partial z}$ in the $\mathrm{MH}$ compared to that in the PI had a positive contribution to the weakening in the EK term. As for $\beta_{w}$, it represents the response of anomalous upwelling (hereafter $w^{\prime}$ ) to $\tau_{x}^{\prime}$. In response to a unit eastward $\tau_{x}^{\prime}$, the response of $w^{\prime}$ would exhibit anomalous downwelling $\left(w^{\prime}<0\right)$ in the eastern equatorial Pacific, because the response of $u^{\prime}$ exhibited its maximum in the central equatorial Pacific (Fig. 11a, b), the gradual decrease of eastward $u^{\prime}$ would induce surface water convergence and hence anomalous downwelling in the eastern equatorial Pacific. Since the response of $u^{\prime}$ to $\tau_{x}^{\prime}$ became weakened in the MH compared to that in the PI, the response of $w^{\prime}$ to $\tau_{x}^{\prime}$, accordingly, became weakened in the MH (see the shading in Fig. 11c). Here, the relationship between the change in $u^{\prime}$ response and that in $w^{\prime}$ response is consistent with the findings of previous studies (e.g., Chen et al. 2015, 2017; Wang et al. 2018), in which the authors pointed out that the change in $w^{\prime}$ response to $\tau_{x}^{\prime}$ in the equatorial region was generally coherent with the change in $u^{\prime}$ response to $\tau_{x}^{\prime}$.

To sum up, the process-oriented analyses above documented that the weakening in the three oceanic dynamic terms primarily arose from the weakened $D^{\prime}$ response to $\tau_{x}^{\prime}$ (i.e., $\beta_{h}$ ), which was induced by the enhanced mean STC in the MH compared to that in the PI.

\section{Conclusions and discussion}

\subsection{Conclusions}

In this study, a CGCM of FGOALS-g2 that exhibits excellent skill in simulating the present-day ENSO was used to investigate the drivers of the reduced ENSO variability in the MH compared to that in the PI. In the MH simulation by the FGOALS-g2, a pronounced decrease of the ENSO intensity was found compared to that in the PI simulation. Through quantifying ENSO-related dynamic and thermodynamic coupling processes in both PI and MH simulations, we demonstrated that the reduced ENSO variance during the $\mathrm{MH}$ arose from the weakening in the oceanic dynamic terms, while the thermodynamic term change had a negative contribution to the reduced ENSO intensity. Among the three oceanic dynamic terms that had positive contributions, the change in the TH term played a leading role in causing the decrease in ENSO intensity, and the changes in the ZA term and the EK term played secondary and tertiary roles, respectively. Considering that these three oceanic dynamic terms involved several components (i.e., the corresponding mean state part and air-sea feedback sub-processes), we further isolated the relative contribution from each component 
to the change of an individual oceanic dynamic term, based on a total derivative relationship. Results showed that the reductions in the TH, ZA, and EK terms were mainly attributed to the weakening in the associated air-sea feedback sub-processes, including $\beta_{h}, a_{h}, \beta_{u}$, and $\beta_{w}$. The weakening in these air-sea feedback sub-processes were linked to the mean state changes in the MH compared to those in the PI, including the enhanced mean STC and the more diffused stratification of upper-ocean mean temperature in the $\mathrm{MH}$. The specific logic chains for the reduced ENSO intensity in the $\mathrm{MH}$ and the changes in the key air-sea feedbacks are stated as follows.

1. Our calculation showed a more negative BJ index in the MH compared to that in the PI, which explains the reduced ENSO variance in the MH. Physically, the more negative $\mathrm{BJ}$ index in the $\mathrm{MH}$ than in the PI indicates a more stabilized coupled ocean-atmosphere system, which was unfavorable for ENSO's growth rate and hence caused weaker ENSO intensity in the MH than in the PI. The more negative $\mathrm{BJ}$ index in the $\mathrm{MH}$ primarily arose from the weakening in the TH term, followed by those in the ZA and EK terms.

2. The weakened $\mathrm{TH}$ term in the $\mathrm{MH}$ compared to that in the PI mainly arose from the weakening in $\beta_{h}$, and followed by the weakening in $a_{h}$. The reduced $\beta_{h}$ was linked to the enhanced mean STC in the MH. The enhanced mean STC that corresponded to an enhanced surface poleward transport led to a relatively flatter meridional structure of ENSO-related $\tau_{x}^{\prime}$ in the MH compared to that in the PI. This indicates the distribution of $\tau_{x}^{\prime}$ at the equator was relatively weaker in the $\mathrm{MH}$ compared to that in the PI, given the same magnitude of $\tau_{x}^{\prime}$ in the Niño4 region. Consequently, such relatively weaker $\tau_{x}^{\prime}$ at the equator induced the thermocline depth change less effectively in the $\mathrm{MH}$ than in the PI, i.e., the strength of $\beta_{h}$ was weakened in the MH compared to that in the PI. The reduced $a_{h}$ was caused by the more diffusive vertical distribution of mean ocean temperature along the equator in the eastern Pacific in the MH than in the PI.

3. The reduced ZA term in the MH resulted from the decrease in the strength of $\beta_{u}$ in the MH compared to that in the PI. We further revealed that the weakening in $\beta_{u}$ was attributed to the weakening in anomalous zonal geostrophic current, which was also linked to the weakening in $\beta_{h}$ (i.e., $D^{\prime}$ response to $\tau_{x}^{\prime}$ ). The physical linkage of the reduced ZA term in the MH compared to that in the PI can be illustrated as $S S H^{\prime} \sim D^{\prime}-->u_{\mathrm{g}}{ }^{\prime}->u^{\prime}-->\beta_{u^{--}}$ $>$ ZA.

4. The reduced EK term in the MH arose from the weakened strength of $\beta_{w}$, and followed by the decrease in the vertical gradient of mean temperature $\left(\frac{\partial \bar{T}}{\partial z}\right)$. Because of the mass continuation, the weakened response of $w^{\prime}$ to $\tau_{x}^{\prime}\left(\right.$ i.e., $\beta_{w}$ ) was linked to the aforementioned weakened response of $u_{-}^{\prime}$ to $\tau_{x}^{\prime}$ (i.e., $\beta_{u}$ ) in the $\mathrm{MH}$. Additionally, the decrease in $\frac{\partial \bar{T}}{\partial z}$ was due to the aforementioned more dif-

fusive vertical distribution of mean ocean temperature in the $\mathrm{MH}$.

\subsection{Discussion}

It is worth mentioning that several recent studies also explored the ENSO intensity change in the MH through analyzing model simulations. The discussion below compares this study with other modeling studies. Some previous studies (Liu et al. 2002; Timmermann et al. 2007) proposed that the reduced ENSO variability change may be related to the enhanced seasonal cycle over tropical Pacific, and suggested that there is an inverse correlation between the strength of the seasonal cycle and the magnitude of the interannual variability. However, the aforementioned argument is still debated. For example, Braconnot et al. (2012) found that both the ENSO amplitude and seasonal cycle amplitude were weakened during $\mathrm{MH}$ in their modeling study. Moreover, the ENSO reconstruction records did not show such a reversed change relationship (EmileGeay et al. 2016). In the present study, we found that the seasonal cycle in eastern Pacific SST was also reduced in the MH simulation by FGOALS-g2 (figure not shown). This indicates that our current results (i.e., both the ENSO amplitude and seasonal cycle amplitude were weakened in $\mathrm{MH}$ compared to PI) seem to support the finding by Braconnot et al. (2012) and Emile-Geay et al. (2016). Therefore, it is difficult to tell whether there is a robust relationship between ENSO amplitude change and seasonal cycle change. One possible reason is that there may be some other factors which are more essential in determining the ENSO amplitude change in MH, even though the reversed change relationship works. This is why we need to quantify the dynamic and thermodynamic air-sea feedback processes that contribute to ENSO variability in PI and $\mathrm{MH}$, and then identify the key drivers and investigate the causes for the key drivers.

Additionally, a study by An and Bong (2017) suggested that the reduced ENSO variability was caused by the intensified Pacific meridional overturning circulation, because the latter induced a strengthening in the dynamic damping by the MA term. Consistently, we also found the mean STC was strengthened and the MA term was enhanced in the MH compared to those in the PI. However, our modeling result showed that the influence of the change in the MA term on the changes in the BJ index and the ENSO intensity was negligible. Another difference between their study and ours lies in that their analyses were based on several 
model mean results, but those models did not include the FGOALS-g2 used in this study. Although their results were built on more model simulations, they found the spatial pattern of the change in the SSTA variance derived from their model mean results did not match the observed pattern, and the decrease of ENSO intensity was only $13 \%$, which was lower than that indicated by the paleo-proxies (ranging from 20 to $79 \%$ ). They noted this discrepancy might be associated with the ENSO simulation biases in the CGCMs they used. This implies that the conclusions derived from the CGCM that has relatively better ENSO simulation skills may be more credible. Nonetheless, it is admitted that the current study was built on a single CGCM; to reduce the uncertainty and verify our conclusions in this study, we may need to conduct a further study with more CGCMs in future.

Acknowledgements We would like to thank three anonymous reviewers for insightful suggestions and comments. This work was jointly supported by the National Key Research and Development Program on Monitoring, Early Warning and Prevention of Major Natural Disaster (2018YFC1506002), the National Natural Science Foundation of China (No. 41705059, No. 41630423, No. 41420104002, and No. 41606011), NSF AGS-1565653, National 973 project 2015CB453200, the Startup Foundation for Introducing Talent of NUIST, NSFC-Shandong Joint Fund for Marine Science Research Centers (U1606405), the LASG Open Project, the open fund of State Key Laboratory of Loess and Quartary Geology, and the Priority Academic Program Development of Jiangsu Higher Education Institutions (PAPD). This is SOEST contribution number 10473, IPRC contribution number 1350, and ESMC number 239 .

Open Access This article is distributed under the terms of the Creative Commons Attribution 4.0 International License (http://creativeco mmons.org/licenses/by/4.0/), which permits unrestricted use, distribution, and reproduction in any medium, provided you give appropriate credit to the original author(s) and the source, provide a link to the Creative Commons license, and indicate if changes were made.

\section{Appendix}

Based on the total derivative relationship, the change in a term [which equals to $(A * B * C * D)$ ] may be written as:

$$
\begin{aligned}
& \mathrm{d}(A * B * C * D)=d(A) *(B * C * D) \\
& \quad+d(B) *(A * C * D)+d(C) *(A * B * D) \\
& \quad+d(D) *(A * B * C)+d(A) * d(B) * C * D \\
& \quad+d(A) * d(C) * B * D+d(A) * d(\mathrm{D}) * B * C \\
& \quad+d(B) * d(C) * A * D+d(B) * d(D) * A * C \\
& \quad+d(C) * d(D) * A * B+d(A) * d(B) * d(C) * D \\
& \quad+d(A) * d(B) * d(D) * C+d(A) * d(C) * d(D) * B \\
& \quad+d(B) * d(C) * d(D) * A+d(A) * d(B) * d(C) * \mathrm{~d}(D)
\end{aligned}
$$

in which $A, B, C$, and $D$ denote the values derived from the PI simulation, and $d()$ represents the difference between PI and MH simulations (MH simulation minus PI simulation). Then, Eq. (10) can be further simplified as follows,

$$
\begin{aligned}
\mathrm{d}(A & * B * C * D)=d(A) *(B * C * D)+d(B) *(A * C * D) \\
& +d(C) *(A * B * D)+d(D) *(A * B * C)+R \\
\mathrm{R}= & d(A) * d(B) * C * D+d(A) * d(C) * B * D \\
& +d(A) * d(\mathrm{D}) * B * C+d(B) * d(C) * A * D \\
& +d(B) * d(D) * A * C+d(C) * d(D) * A * B \\
& +d(A) * d(B) * d(C) * D+d(A) * d(B) * d(D) * C \\
& +d(A) * d(C) * d(D) * B+d(B) * d(C) * d(D) * A \\
& +d(A) * d(B) * d(C) * \mathrm{~d}(D)
\end{aligned}
$$

where $R$ indicates the contribution by the covariant changes. Following the above equations, the change of the TH term may be written as follows,

$$
\begin{aligned}
& \mathrm{d}(T H)=d\left(\mu_{a} * \beta_{h} * a_{h} * \frac{\bar{W}}{H_{1}}\right) \\
& =d\left(\mu_{a}\right) *\left(\beta_{h} * a_{h} * \frac{\bar{W}}{H_{1}}\right)+d\left(\beta_{h}\right) *\left(\mu_{a} * a_{h} * \frac{\bar{W}}{H_{1}}\right) \\
& +d\left(a_{h}\right) *\left(\mu_{a} * \beta_{h} * \frac{\bar{W}}{H_{1}}\right)+d\left(\frac{\bar{W}}{H_{1}}\right) *\left(\mu_{a} * \beta_{h} * a_{h}\right)+R_{1}
\end{aligned}
$$

where $d(\mathrm{TH})$ denotes the TH term change, the first three terms on the right-hand side of Eq. (13) indicate the contributions by the corresponding air-sea feedback changes to $d(\mathrm{TH})$, the fourth term on the right-hand side indicates the contribution by the corresponding mean state change to $d(\mathrm{TH})$, and $R_{l}$ indicates the contribution by the covariant changes of both the mean state and the air-sea feedbacks.

Likewise, applying the total derivative relationship to the ZA term and EK term, which are involved with three factors, we may have

$$
\begin{aligned}
\mathrm{d}(\mathrm{ZA})= & d\left(\mu_{a} * \beta_{u} *\left(-\frac{\partial \bar{T}}{\partial x}\right)\right)=d\left(\mu_{a}\right) *\left(-\frac{\partial \bar{T}}{\partial x} * \beta_{u}\right) \\
& +d\left(\beta_{u}\right) *\left(-\frac{\partial \bar{T}}{\partial x} * \mu_{a}\right)+\mathrm{d}\left(-\frac{\partial \bar{T}}{\partial x}\right) *\left(\mu_{a} * \beta_{h}\right)+R_{2}
\end{aligned}
$$

where $d(\mathrm{ZA})$ denotes the ZA term change, the first two terms on the right-hand side of Eq. (14) indicate the contributions by the corresponding air-sea feedback changes to $d(\mathrm{ZA})$, the third term on the right-hand side indicates the contribution by the corresponding mean state change to $d(\mathrm{ZA})$, and $R_{2}$ indicates the contribution by the covariant changes of both the mean state and the air-sea feedbacks. 


$$
\begin{aligned}
\mathrm{d}(\mathrm{EK})= & d\left(\mu_{a} * \beta_{w} *\left(-\frac{\partial \bar{T}}{\partial z}\right)\right)=d\left(\mu_{a}\right) *\left(-\frac{\partial \bar{T}}{\partial z} * \beta_{w}\right) \\
& +d\left(\beta_{w}\right) *\left(-\frac{\partial \bar{T}}{\partial z} * \mu_{a}\right)+\mathrm{d}\left(-\frac{\partial \bar{T}}{\partial z}\right) *\left(\mu_{a} * \beta_{w}\right)+R_{3}
\end{aligned}
$$

where $d(\mathrm{EK})$ denotes the EK term change, the first two terms on the right-hand side of Eq. (15) indicate the contributions by the corresponding air-sea feedback changes to $d(\mathrm{EK})$, the third term on the right-hand side indicates the contribution by the corresponding mean state change to $d(\mathrm{EK})$, and $R_{3}$ indicates the contribution by the covariant changes of both the mean state and the air-sea feedbacks.

\section{References}

An S-I, Bong H (2017) Feedback process responsible for the suppression of ENSO activity during the mid-Holocene. Theor Appl climatology:1-12 https://doi.org/10.1007/s00704-017-2117-6

An S-I, Choi J (2014) Mid-Holocene tropical Pacific climate state, annual cycle, and ENSO in PMIP2 and PMIP3. Clim Dyn 43:957-970. https://doi.org/10.1007/s00382-013-1880-z

An S-I, Jin F-F (2001) Collective role of thermocline and zonal advective feedbacks in the ENSO mode*. J Clim 14:3421-3432

Bellenger H, Guilyardi É, Leloup J, Lengaigne M, Vialard J (2014) ENSO representation in climate models: from CMIP3 to CMIP5. Clim Dyn 42:1999-2018

Braconnot P et al. (2007b) Results of PMIP2 coupled simulations of the Mid-Holocene and last glacial maximum-part 2: feedbacks with emphasis on the location of the ITCZ and mid- and high latitudes heat budget. Clim Past 3:279-296. https://doi. org/10.5194/cp-3-279-2007

Braconnot $\mathrm{P}$ et al. (2007b) Results of PMIP2 coupled simulations of the Mid-Holocene and last glacial maximum-PART 1: experiments and large-scale features. Clim Past 3:261-277. https://doi. org/10.5194/cp-3-261-2007

Braconnot P, Luan Y, Brewer S, Zheng W (2012) Impact of Earth's orbit and freshwater fluxes on Holocene climate mean seasonal cycle and ENSO characteristics. Clim Dyn 38:1081-1092. https ://doi.org/10.1007/s00382-011-1029-x

Brown J, Collins M, Tudhope AW, Toniazzo T (2008) Modelling mid-Holocene tropical climate and ENSO variability: towards constraining predictions of future change with palaeo-data. Clim Dyn 30:19-36. https://doi.org/10.1007/s00382-007-0270-9

Chen L, Yu Y, Sun D-Z (2013) Cloud and water vapor feedbacks to the El Niño warming: are they still biased in CMIP5 models? J Clim 26:4947-4961. https://doi.org/10.1175/jcli-d-12-00575.1

Chen L, Li T, Yu Y (2015) Causes of strengthening and weakening of ENSO amplitude under global warming in four CMIP5 models. J Clim 28:3250-3274. https://doi.org/10.1175/jcli-d-14-00439.1

Chen L, Yu Y, Zheng W (2016) Improved ENSO simulation from climate system model FGOALS-g1.0 to FGOALS-g2. Clim Dyn 47:2617-2634. https://doi.org/10.1007/s00382-016-2988-8

Chen L, Li T, Yu Y, Behera SK (2017) A possible explanation for the divergent projection of ENSO amplitude change under global warming. Clim Dyn 49:3799-3811. https://doi.org/10.1007/ s00382-017-3544-x

Chen L, Wang L, Li T, Sun D-Z (2018a) Contrasting cloud radiative feedbacks during warm pool and cold tongue El Niños.
Sci Online Lett Atmos 14:126-131. https://doi.org/10.2151/ sola.2018-022

Chen L, Sun D-Z, Wang L, Li T (2018b) A further study on the simulation of cloud-radiative feedbacks in the ENSO cycle in the tropical Pacific with a focus on the asymmetry. Asia-Pac J Atmos Sci. https://doi.org/10.1007/s13143-018-0064-5

Chiang JCH, Fang Y, Chang P (2009) Pacific Climate Change and ENSO Activity in the Mid-Holocene. J Clim 22:923-939. https ://doi.org/10.1175/2008jcli2644.1

Clement AC, Seager R, Cane MA (2000) Suppression of El Niño during the Mid-Holocene by changes in the Earth's orbit. Paleoceanography 15:731-737

Cobb KM, Charles CD, Cheng H, Edwards RL (2003) El Niño/ Southern Oscillation and tropical Pacific climate during the last millennium. Nature 424:271. https://doi.org/10.1038/natur e01779

Cobb KM et al (2013) Highly variable El Niño-Southern oscillation throughout the Holocene. Science 339:67-70. https://doi. org/10.1126/science. 1228246

Collins $\mathrm{M}$ et al (2010) The impact of global warming on the tropical Pacific Ocean and El Niño. Nat Geosci 3:391-397

Emile-Geay J et al (2016) Links between tropical Pacific seasonal, interannual and orbital variability during the Holocene. Nature Geosci 9:168-173. https://doi.org/10.1038/ngeo2608

Hua L, Chen L, Rong X, Su J, Wang L, Li T, Yu Y (2018) Impact of atmospheric model resolution on simulation of ENSO feedback processes: a coupled model study. Clim Dyn. https://doi. org/10.1007/s00382-017-4066-2

Hunke EC, Lipscomb WH (2008) CICE: The Los Alamos sea ice model user's manual, version 4. Los Alamos National Laboratory Tech. Rep. LA-CC-06-012, 76

Jin FF, Kim ST, Bejarano L (2006) A coupled-stability index for ENSO. Geophys Res Lett 33:L23708

Karamperidou C, Di Nezio PN, Timmermann A, Jin F-F, Cobb KM (2015) The response of ENSO flavors to mid-Holocene climate: proxy interpretation. Paleoceanography 30: https://doi. org/10.1002/2014pa002742

Kim ST, Jin F-F (2011a) An ENSO stability analysis. Part I: results from a hybrid coupled model. Clim Dyn 36:1593-1607

Kim ST, Jin F-F (2011b) An ENSO stability analysis. Part II: results from the twentieth and twenty-first century simulations of the CMIP3 models. Clim Dyn 36:1609-1627

Kim ST, Cai W, Jin F-F, Santoso A, Wu L, Guilyardi E, An S-I (2014a) Response of El Nino sea surface temperature variability to greenhouse warming. Nat Clim Change 4:786-790. https://doi. org/10.1038/nclimate2326

Kim ST, Cai W, Jin F-F, Yu J-Y (2014b) ENSO stability in coupled climate models and its association with mean state. Clim Dyn 42:3313-3321

Kitoh A, Murakami S (2002) Tropical Pacific climate at the mid-Holocene and the Last Glacial Maximum simulated by a coupled ocean-atmosphere general circulation model. Paleoceanography 17:1047. https://doi.org/10.1029/2001pa000724

Koutavas A, Joanides S (2012) El Niño-Southern oscillation extrema in the Holocene and last glacial maximum. Paleoceanography 27:PA4208. https://doi.org/10.1029/2012pa002378

Li T (1997) Phase transition of the El Niño-Southern oscillation: a stationary SST mode. J Atmos Sci 54:2872-2887

Li L et al (2013a) The flexible global ocean-atmosphere-land system model, Grid-point Version 2: FGOALS-g2. Adv Atmos Sci 30:543-560

Li L et al (2013b) Evaluation of grid-point atmospheric model of IAP LASG version 2 (GAMIL2). Adv Atmos Sci 30:855-867

Li L, Wang B, Zhang GJ (2015a) The role of moist processes in shortwave radiative feedback during ENSO in the CMIP5 
models. J Clim 28:9892-9908 doi. https://doi.org/10.1175/ JCLI-D-15-0276.1

Li Y, Li J-P, Zhang W, Zhao X, Xie F, Zheng F (2015b) Ocean dynamical processes associated with the tropical Pacific cold tongue mode. J Geophys Res: Oceans 120:6419-6435. https://doi. org/10.1002/2015jc010814

Li Y, Li J-P, Zhang W, Chen Q, Feng Juan Z, Wang W, Zhou X (2017) Impacts of the tropical pacific cold tongue mode on ENSO diversity under global warming. J Geophys Res: Oceans 122:8524 8542. https://doi.org/10.1002/2017jc013052

Liu Z (2002) A simple model study of ENSO suppression by external periodic forcing. J Clim 15:1088-1098 https://doi. org/10.1175/1520-0442(2002)015\%3C1088:asmsoe\%3E2.0.co;2

Liu Z, Kutzbach J, Wu L (2000) Modeling climate shift of El Nino variability in the Holocene. Geophys Res Lett 27:2265-2268. https://doi.org/10.1029/2000gl011452

Liu H, Lin P, Yu Y, Zhang X (2012) The baseline evaluation of LASG/ IAP climate system ocean model (LICOM) version 2. Acta Meteorologica Sinica 26:318-329

Liu Z, Lu Z, Wen X, Otto-Bliesner BL, Timmermann A, Cobb KM (2014) Evolution and forcing mechanisms of El Nino over the past 21,000 years. Nature 515:550-553. https://doi.org/10.1038/ nature 13963

Lloyd J, Guilyardi E, Weller H (2012) The role of atmosphere feedbacks during ENSO in the CMIP3 models. The Shortwave Flux Feedback, Part

Luan Y, Braconnot P, Yu Y, Zheng W, Marti O (2012) Early and mid-Holocene climate in the tropical Pacific: seasonal cycle and interannual variability induced by insolation changes. Clim Past 8:1093-1108. https://doi.org/10.5194/cp-8-1093-2012

Luan Y, Braconnot P, Yu Y, Zheng W (2015) Tropical Pacific mean state and ENSO changes: sensitivity to freshwater flux and remnant ice sheets at $9.5 \mathrm{ka}$ BP. Clim Dyn 44:661-678. https://doi. org/10.1007/s00382-015-2467-7

Lübbecke JF, McPhaden MJ (2014) Assessing the 21st century shift in ENSO variability in terms of the Bjerknes stability index. $\mathbf{J}$ Clim 27:2577-2587

McCreary JP Jr, Lu P (1994) Interaction between the subtropical and equatorial ocean circulations: the subtropical cell. J Phys Oceanogr 24:466-497

McGregor HV, Gagan MK (2004) Western Pacific coral $\delta 180$ records of anomalous Holocene variability in the El NiñoSouthern Oscillation. Geophys Res Lett 31:L11204. https://doi. org/10.1029/2004g1019972

McGregor HV, Fischer MJ, Gagan MK, Fink D, Phipps SJ, Wong H, Woodroffe CD (2013) A weak El Nino/Southern Oscillation with delayed seasonal growth around 4,300 years ago. Nature Geosci 6:949-953. https://doi.org/10.1038/ngeo1936

McPhaden MJ, Zebiak SE, Glantz MH (2006) ENSO as an integrating concept in earth science. Science 314:1740-1745. https://doi. org/10.1126/science. 1132588

Oleson KW et al (2004) Technical description of the community land model (CLM). In: Tech. Rep. NCAR/TN-461 + STR, National Center for Atmospheric Research, Boulder, $174 \mathrm{pp}$

Otto-Bliesner BL, Brady EC, Shin S-I, Liu Z, Shields C (2003) Modeling El Niño and its tropical teleconnections during the last glacial-interglacial cycle. Geophys Res Lett 30:2198. https://doi. org/10.1029/2003gl018553

Philander SG (1990) El Niño, La Niña, and the Southern Oscillation. Academic Press, London, 293 pp
Roberts WHG, Battisti DS, Tudhope AW (2014) ENSO in the MidHolocene according to CSM and HadCM3. J Clim 27:1223-1242 doi. https://doi.org/10.1175/JCLI-D-13-00251.1

Su J, Zhang R, Li T, Rong X, Kug J, Hong C-C (2010) Causes of the El Niño and La Niña amplitude asymmetry in the equatorial eastern Pacific. J Clim 23:605-617

Su J, Li T, Zhang R (2014) The initiation and developing mechanisms of central Pacific El Niños. J Clim 27:4473-4485

Sun D-Z, Yu Y, Zhang T (2009) Tropical water vapor and cloud feedbacks in climate models: a further assessment using coupled simulations. J Clim 22:1287-1304. https://doi.org/10.1175/2008jcli22 67.1

Tian Z, Jiang D (2013) Mid-Holocene ocean and vegetation feedbacks over East Asia. Clim Past 9:2153-2171. https://doi.org/10.5194/ cp-9-2153-2013

Tian Z, Li T, Jiang D, Chen L (2017) Causes of ENSO weakening during the Mid-Holocene. J Clim 30:7049-7070. https://doi. org/10.1175/jcli-d-16-0899.1

Tian Z, Li T, Jiang D (2018) Strengthening and westward shift of the tropical pacific walker circulation during the Mid-Holocene: PMIP simulation results. J Clim 31:2283-2298. https://doi. org/10.1175/jcli-d-16-0744.1

Timmermann A, Lorenz SJ, An S-I, Clement A, Xie S-P (2007) The Effect of orbital forcing on the mean climate and variability of the tropical Pacific. J Clim 20:4147-4159. https://doi.org/10.1175/ jcli4240.1

Trenberth KE, Branstator GW, Karoly D, Kumar A, Lau N-C, Ropelewski C (1998) Progress during TOGA in understanding and modeling global teleconnections associated with tropical sea surface temperatures. J Geophys Res: Oceans 103:14291-14324. https://doi.org/10.1029/97jc01444

Tudhope AW et al (2001) Variability in the El Niño-Southern oscillation through a glacial-interglacial cycle. Science 291:1511-1517. https://doi.org/10.1126/science.1057969

Wang Y, Luo Y, Lu J, Liu F (2018) Changes in ENSO amplitude under climate warming and cooling. Clim Dyn. https://doi.org/10.1007/ s00382-018-4224-1

Woodroffe CD, Gagan MK (2000) Coral microatolls from the central Pacific record Late Holocene El Niño. Geophys Res Lett 27:15111514. https://doi.org/10.1029/2000gl011407

Zhang W, Jin FF (2012) Improvements in the CMIP5 simulations of ENSO-SSTA meridional width. Geophys Res Lett 39

Zhang W, Li J, Jin FF (2009) Spatial and temporal features of ENSO meridional scales. Geophys Res Lett 36 https://doi. org/10.1029/2009GL038672

Zhang W, Jin F-F, Zhao J-X, Li J (2013) On the Bias in Simulated ENSO SSTA Meridional Widths of CMIP3 Models. J Clim 26:3173-3186

Zheng W, Yu Y (2013) Paleoclimate simulations of the mid-Holocene and last glacial maximum by FGOALS. Adv Atmos Sci 30:684698. https://doi.org/10.1007/s00376-012-2177-6

Zheng W, Braconnot P, Guilyardi E, Merkel U, Yu Y (2008) ENSO at 6 $\mathrm{ka}$ and $21 \mathrm{ka}$ from ocean-atmosphere coupled model simulations. Clim Dyn 30:745-762

Zhou T, Yu Y, Liu Y, Wang B (2014) Flexible global ocean-atmosphere-land system model: a modeling tool for the climate change research community. Springer, New York. https://doi. org/10.1007/978-3-642-41801-3 